
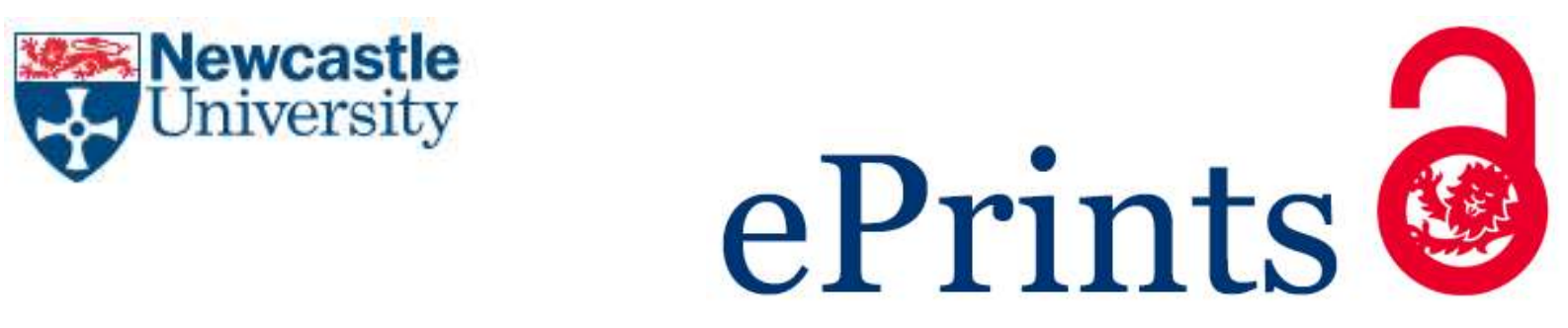

Lai J, Klein M, Chakraborty N. Assessment of algebraic Flame Surface Density closures in the context of Large Eddy Simulations of head-on quenching of turbulent premixed flames. Combustion Science and Technology 2017

\title{
Copyright:
}

This is an Accepted Manuscript of an article published by Taylor \& Francis in Combustion Science and Technology on $30^{\text {th }}$ June 2017, available online: http://dx.doi.org/10.1080/00102202.2017.1347161

Date deposited:

$13 / 07 / 2017$

Embargo release date:

30 June 2018

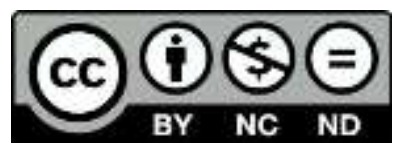

This work is licensed under a

Creative Commons Attribution-NonCommercial-NoDerivatives 4.0 International licence 


\section{Assessment of algebraic Flame Surface Density closures in the context of Large Eddy Simulations of head-on quenching of turbulent premixed flames}

Jiawei Lai*, Markus Klein**, Nilanjan Chakraborty*a

*School of Mechanical \& Systems Engineering, Newcastle University, Newcastle-Upon-Tyne, NE1 7RU, UK

** Department of Aerospace Engineering, Universität der Bundeswehr München, Neubiberg, 85577, Germany

*a Corresponding author

Tel.: +441912083570

Fax: +44 1912088600

E-mail: nilanjan.chakraborty@ncl.ac.uk

Keywords: Flame Surface Density, Large Eddy Simulation, Premixed combustion, Head-on quenching, Direct Numerical Simulation 


\section{ABSTRACT}

The applicability of algebraic Large Eddy Simulation (LES) closures of Flame Surface Density (FSD) $\Sigma_{g e n}$ for head-on quenching of premixed turbulent flames by an isothermal inert wall has been assessed using three-dimensional DNS data for different values of root-mean-square turbulent velocity fluctuation, Damköhler and Karlovitz numbers. An algebraic FSD closure, which has been reported to perform relatively satisfactorily among several available models, has been considered for this analysis alongside a model which has recently been used for LES of flame-wall interaction. The applicability of previously proposed near-wall damping factors for flame surface wrinkling and consumption rate in the context of Reynolds Averaged Navier Stokes (RANS) simulations has also been assessed for LES based on the current a-priori DNS analysis. It has been found that existing models considered for this analysis do not predict the near-wall behaviour of the FSD accurately for all cases considered here. Furthermore, the widely used expression $\rho_{0} S_{L} \Sigma_{\text {gen }}$ (where $\rho_{0}$ and $S_{L}$ are the unburned gas density and the laminar burning velocity respectively) has been found to overpredict the combined reaction rate and molecular diffusion term in the near-wall region but the agreement between these terms gets better away from the wall. However, $\rho_{0} S_{L}$ does not sufficiently capture the local behaviour of the density-weighted surface filtered displacement speed so the correlation coefficient between $\rho_{0} S_{L} \Sigma_{g e n}$ and the combined reaction rate and molecular diffusion term remains much smaller than unity. It has been found that the damping factors proposed for RANS are not suitable for LES, and they severely damp the near-wall magnitudes of FSD and the combined reaction rate and molecular diffusion term and lead to significant underpredictions. Based on this a-priori analyses new near-wall modifications to the generalised FSD and the combined reaction and molecular diffusion term have been proposed in the context of LES, which have been found to capture both qualitative and quantitative trends obtained from DNS data. 


\section{INTRODUCTION}

Flame Surface Density (FSD) is often used for the closure of filtered/averaged chemical reaction rate in premixed turbulent combustion. In the context of this methodology, modelling of filtered/averaged reaction rate translates to the closure of the flame surface area to volume ratio (Candel and Poinsot, 1990). The FSD itself is an unclosed quantity, which is modelled in the context of both Reynolds Averaged Navier-Stokes (RANS) and Large Eddy Simulations (LES) by either an algebraic expression in terms of known quantities (Boger et al., 1998; Butz et al., 2015; Cant and Bray, 1988; Chakraborty and Klein, 2008; Fureby, 2005; Katragadda et al., 2012a,b; Keppeler et al., 2014; Klein et al., 2016; Ma et al., 2013, 2014a,b) or by solving a modelled transport equation (Cant et al., 1990; Candel et al., 1990;Chakraborty and Cant, 2007, 2009; Hawkes and Cant, 2000, 2001; Hernandez-Perez et al., 2011; Katragadda et al., 2014; Ma et al., 2014b; Reddy and Abraham, 2012). It is often preferable to use the algebraic FSD closure in comparison to the transported FSD closure because of the computational economy, in the context of LES. Interested readers are referred to (Chakraborty and Klein, 2008; Katragadda et al., 2012a,b; Keppeler et al., 2014; Klein et al., 2016; Ma et al., 2013) and references therein for an extensive review of algebraic FSD closures. It is worth noting that most FSD models (Cant and Bray, 1988; Cant et al., 1990; Candel et al., 1990) have been proposed and most Large Eddy Simulations (LES) (Boger et al., 1998; Butz et al., 2015; Chakraborty and Cant, 2007, 2009; Chakraborty and Klein, 2008; Fureby, 2005; Hawkes and Cant, 2000, 2001; Hernandez-Perez et al., 2011; Katragadda et al., 2012a,b, 2014; Ma et al., 2013, 2014a,b; Keppeler et al., 2014; Klein et al., 2016; Reddy and Abraham, 2012) have been conducted for flows away from the wall, and it remains to be seen if these models perform satisfactorily in the near-wall region. 
The interactions between flame and wall influence the efficiency of combustion systems, formation of pollutants (e.g. unburned hydrocarbons), wall cooling for safe operation and overall durability of combustors (Heywood, 1988; Poinsot and Veynante, 2001). Moreover, the operation and durability of small and micro-combustors are severely limited by flame quenching by the cold wall due to high heat transfer through the wall because of high surface area to volume ratio. Moreover, flame propagation in turbulent boundary layers has been identified as one of the mechanisms of flashback into the mixing zone from the combustion chamber (Poinsot and Veynante, 2001). In spite of its importance, flame wall-interaction (FWI) has not been analysed in detail because of its complexity, and the difficulties that it offers for both experimental and computational analyses. In most practical combustion devices, the burned gas temperature can attain values in excess of $2000 \mathrm{~K}$, whereas the wall temperature of the combustor is often kept in the range of $800-1000 \mathrm{~K}$ because of the cooling process so that structural integrity is maintained. In combustors of IC engines and gas turbines, flame quenching by cold walls leads to unburned hydrocarbons, which, in combination with heat losses to the wall, negatively affects the performance, efficiency and pollutant emission characteristics of the engine. It has been shown previously by several researchers (Poinsot $e t$ al., 1993; Bruneaux et al., 1996,1997; Alshaalan and Rutland, 1998,2002; Gruber et al., 2010, 2012; Dabireau et al., 2013; Lai et al., 2016a-c) that the flame quenches close to the wall due to heat loss through the wall, which leads to significant drops in the flame surface area and the reaction rate in the near-wall region. A few analyses (Bruneaux et al., 1997; Alshaalan and Rutland, 1998) concentrated on the near-wall modifications to the FSD based reaction rate closures in the context of RANS but the performance of FSD closures in the near-wall region and the necessity of near-wall damping factors are yet to be assessed in detail in the context of LES. A recent LES analysis (Keppeler et al., 2012) used the wall damping factors, which were previously proposed in the context of RANS. The present analysis focuses on the assessment 
of algebraic FSD closures in the near-wall region based on Direct Numerical Simulations (DNS) data of head-on quenching of statistically planar flames by inert isothermal walls. For the purpose of the present analysis, an algebraic FSD model proposed by Fureby (2005) (which has been shown to provide satisfactory prediction for premixed turbulent flames based on both a-priori DNS (Chakraborty and Klein, 2008; Katragadda et al., 2012a,b, 2014; Klein et al., 2016) and a-posteriori LES assessments (Butz et al., 2015; Ma et al., 2013, 2014a,b)) has been considered along with a model proposed by Keppeler et al. $(2012,2014)$, which was later modified for FWI. In this respect, the main objectives of this paper are:

1. To assess the near-wall performances of the algebraic FSD models which are either shown to perform well away from the wall in previous analyses or proposed previously for FWI.

2. To assess if the near-wall damping factors of reaction rate and FSD closures, which were originally proposed in the context of RANS, remain valid also for LES.

3. To identify an algebraic model expression for FSD which is valid both in the near-wall region as well as away from the wall.

The rest of the paper will be organised as follows. The mathematical background and numerical implementation pertaining to this analysis are presented in the next two sections. This will be followed by the presentation of results and its subsequent discussion. The main findings will be summarised and conclusions will be drawn in the final section of this paper.

\section{MATHEMATICAL BACKGROUND}

The present analysis uses a single step generic chemical reaction (i.e. Reactants $\rightarrow$ Products) so that an extensive parametric analysis can be conducted without an exorbitant computational cost because three-dimensional detailed chemical simulations are often extremely expensive (Chen et al., 2009) for this purpose. Several previous analyses (Poinsot $e t$ al., 1993; Bruneaux et al., 1996,1997; Alshaalan and Rutland, 1998, 2002) contributed 
significantly to the fundamental understanding of flame-wall interaction using simple chemistry DNS and the same approach has been adopted here. Interested readers are referred to Poinsot et al. (1993), Bruneaux et al. (1996,1997), and Alshaalan and Rutland (1998, 2002) for the chemical mechanism considered here. It was demonstrated by Poinsot et al. (1993) and Lai and Chakraborty (2016a,b) that the wall heat flux and quenching distance can be adequately captured using simple single step chemistry. In the context of simple chemistry, the reaction progress variable $c$ is defined in terms of a reactant mass fraction $Y_{R}$ as:

$$
c=\frac{\left(Y_{R 0}-Y_{R}\right)}{\left(Y_{R 0}-Y_{R \infty}\right)}
$$

where subscripts 0 and $\infty$ are used to refer to the values in the unburned and fully burned gases respectively. The transport equation of $c$ is given by:

$$
\frac{\partial(\rho c)}{\partial t}+\frac{\partial\left(\rho u_{j} c\right)}{\partial x_{j}}=\frac{\partial}{\partial x_{j}}\left(\rho D \frac{\partial c}{\partial x_{j}}\right)+\dot{\omega}
$$

where $u_{j}, D$ and $\dot{\omega}$ are the gas density, $\mathrm{j}^{\text {th }}$ component of velocity vector, progress variable diffusivity and reaction rate respectively. On filtering eq. 2 one obtains:

$$
\frac{\partial(\bar{\rho} \tilde{c})}{\partial t}+\frac{\partial\left(\bar{\rho} \widetilde{u}_{j} \tilde{c}\right)}{\partial x_{j}}=\overline{\frac{\partial}{\partial x_{j}}\left(\rho D \frac{\partial c}{\partial x_{J}}\right)}+\overline{\dot{\omega}}-\frac{\partial\left(\overline{\rho u_{j} c}-\bar{\rho} \widetilde{u}_{j} \tilde{c}\right)}{\partial x_{j}}
$$

where $\bar{q}$ and $\tilde{q}=\overline{\rho q} / \bar{\rho}$ are the LES filtered and Favre-filtered values of a general quantity $q$. The first two terms on the right hand side are often collectively referred to as the filtered reaction-diffusion imbalance term and the last term is the turbulent transport term arising from sub-grid scalar flux $\left(\overline{\rho u_{\jmath} c}-\bar{\rho} \tilde{u}_{j} \tilde{c}\right)$. The filtered reaction-diffusion imbalance and turbulent transport terms are unclosed and need to be modelled in LES simulations. The present work focuses on the modelling of the filtered reaction-diffusion imbalance term. In the context of FSD based closure the filtered reaction-diffusion imbalance term can be modelled in the following manner (Boger et al., 1998):

$$
\overline{\dot{\omega}}+\overline{\nabla \cdot(\rho D \nabla c)}=\overline{\left(\rho S_{d}\right)_{s}} \Sigma_{g e n}
$$


where $S_{d}=(D c / D t) /|\nabla c|, \Sigma_{g e n}=\overline{|\nabla c|}$ are the displacement speed and the generalised FSD, respectively, and $\overline{(q)_{s}}=\overline{q|\nabla c|} / \Sigma_{g e n}$ is the surface-weighted filtered value of $q$. It is evident from eq. 4 that the filtered reaction-diffusion imbalance term can be closed if $\Sigma_{g e n}$ and $\overline{\left(\rho S_{d}\right)_{s}}$ are appropriately modelled. The quantity ${\overline{\left(\rho S_{d}\right)}}_{s}$ is often approximated by $\overline{\left(\rho S_{d}\right)_{s}} \approx \rho_{0} S_{L}$ where $\rho_{0}$ and $S_{L}$ are the unburned gas density and laminar burning velocity respectively (Butz et al., 2015; Hawkes and Cant, 2000,2001; Hernandez-Perez et al., 2011; Ma et al., 2013,2014a,b). However, existing findings based on recent a-priori DNS analyses (Chakraborty and Cant, 2007,2009, 2011; Katragadda et al., 2014; Klein et al., 2016) suggested that $\overline{\left(\rho S_{d}\right)_{s}} \approx \rho_{0} S_{L}$ may not be valid under all conditions. For the current analysis only algebraic closure of FSD will be considered. A number of recent analyses (Butz et al., 2015; Chakraborty and Klein, 2008; Katragadda et al., 2012a,b; Klein et al., 2016; Ma et al., 2013,2014b) indicated that the algebraic FSD closure proposed by Fureby (2005) performs satisfactorily based on a-priori and a-posteriori assessments. The modified Fureby model is given by (Butz et al., 2015; Klein et al., 2016; Ma et al., 2013,2014b):

$$
\Sigma_{g e n}=\left(1+\Gamma \cdot u_{\Delta}^{\prime} / S_{L}\right)^{D_{f}-2}|\nabla \bar{c}|
$$

where the fractal dimension $D_{f}$, and the efficiency function $\Gamma$ are given by (Fureby, 2005):

$$
D_{f}=\frac{2.05}{u_{\Delta}^{\prime} / S_{L}+1}+\frac{2.35}{S_{L} / u_{\Delta}^{\prime}+1} ; \quad \Gamma=0.75 \exp \left[-\frac{1.2}{\left(u_{\Delta}^{\prime} / S_{L}\right)^{0.3}}\right]\left(\frac{\Delta}{\delta_{\mathrm{Z}}}\right)^{2 / 3}
$$

where $\delta_{\mathrm{Z}}=\alpha_{T 0} / S_{L}$ is the Zel'dovich flame thickness with $\alpha_{T 0}$ being the thermal diffusivity in the unburned gas. The sub-grid scale turbulent velocity fluctuation $u_{\Delta}^{\prime}$ is calculated from the DNS data (by explicit filtering of DNS data according to the expression: $u_{\Delta}^{\prime}=\sqrt{2 k_{s g s} / 3}=$ $\sqrt{\left(\widetilde{u_{l} u_{l}}-\tilde{u}_{i} \tilde{u}_{i}\right) / 3}$, where $k_{s g s}=\left(\widetilde{u_{\imath} u_{\imath}}-\tilde{u}_{i} \tilde{u}_{i}\right) / 2$ is the sub-grid turbulent kinetic energy). for the purpose of this analysis, in order to avoid ambiguity arising from different model expressions available for this term. The same approach was followed in several previous 
analyses (Allauddin et al., 2017; Chakraborty and Klein, 2008; Katragadda et al., 2012a,b; Klein et al., 2016; Ma et al., 2013,2014a,b). It is worth noting that the additive contribution of $|\nabla \bar{c}|$ was absent on the right hand side of eq. 5 in the original model formulation. Furthermore, it is useful to note that the analyses (Butz et al., 2015; Chakraborty and Klein, 2008; Katragadda et al., 2012a,b; Klein et al., 2016; Ma et al., 2013,2014b), which reported satisfactory performance of Fureby's model in the past, were carried out in the absence of a wall.

An alternative filtered flame front displacement model particularly developed to represent high-pressure flames has recently been proposed by Keppeler et al. $(2012,2014)$, which was subsequently used for flame-wall interaction. The original version of the FSD model by Keppeler et al. (2014) is given by:

$$
\Sigma_{g e n}=\left(\frac{2.2 \Delta}{\max \left(\delta_{\mathrm{Z}} K a_{\Delta}^{-1 / 2}, 2 \delta_{\mathrm{Z}}\right)}\right)^{D_{f}-2} 4.5 \tilde{c}(1-\tilde{c}) F(\tilde{c})^{-1}|\nabla \tilde{c}|
$$

Here $K a_{\Delta}$ is the sub-grid Karlovitz number, and the fractal dimension $D_{f}$ is expressed as (Keppeler et al., 2012,2014):

$$
K a_{\Delta}=\left(\frac{u_{\Delta}^{\prime}}{s_{L}^{0}}\right)^{\frac{3}{2}}\left(\frac{\Delta}{\delta_{\mathrm{Z}}}\right)^{-\frac{1}{2}} ; \quad D_{f}=\frac{8 / 3 K a_{\Delta}+2 C_{D}}{K a_{\Delta}+C_{D}} ; C_{D}=0.03
$$

The function $F(\tilde{c})$ can be approximated as $F(\tilde{c})=0.9952-2.8181(\tilde{c}-0.5)^{2}-4.3072(\tilde{c}-$ $0.5)^{4}$. Further details on the derivation of this model can be found elsewhere (Keppeler et al., 2012,2014) and thus are not discussed here.

Alshaalan and Rutland (1998) proposed a damping factor $Q_{A}$ for the prediction of FSD in the context of RANS in order to account for the reduction of flame surface area in the near-wall region due to flame quenching. This damping factor was considered by Keppeler et al. (2012) for the purpose of LES simulations where it takes the following form: 


$$
Q_{A}=\left[1+48\left(\tilde{c}_{w}-\tilde{T}_{w}\right)\right] \exp \left\{-\beta[\tau(\tilde{c}-\tilde{T}) /((1+\tau \tilde{T})(1+\tau \tilde{c}))]^{0.25}\right\}
$$

where $T=\left(\widehat{T}-T_{0}\right) /\left(T_{a d}-T_{0}\right), \tau=\left(T_{a d}-T_{0}\right) / T_{0}$ and $\beta=T_{a c}\left(T_{a d}-T_{0}\right) / T_{a d}^{2}$ are nondimensional temperature, heat release parameter and Zel'dovich number respectively with $\widehat{T}, T_{0}, T_{a c}$ and $T_{a d}$ being the instantaneous dimensional, unburned gas, activation and adiabatic flame temperatures respectively. The subscript ' $w$ ' is used here to refer to values at the wall.

Bruneaux et al. (1997) indicated that $\langle\nabla \cdot(\rho D \nabla c)+\dot{\omega}\rangle_{R}=\rho_{0} S_{L}\langle\nabla c\rangle_{R}$ (where $\langle q\rangle_{R}$ is the Reynolds averaged value of a general quantity $q$ ) does not remain valid and $\rho_{0} S_{L}\langle\nabla c\rangle_{R}$ overpredicts $\langle\nabla \cdot(\rho D \nabla c)+\dot{\omega}\rangle_{R}$ in the near-wall region because of reduced burning rate due to flame quenching. Bruneaux et al. (1997) suggested a damping function $Q_{B}$ so that $\rho_{0} Q_{B} S_{L}\langle\nabla c\rangle_{R}$ is supposed to predict $\langle\nabla \cdot(\rho D \nabla c)+\dot{\omega}\rangle_{R}$ in the context of RANS. It is worth noting that $Q_{B}$ damps the consumption rate in the near-wall region. Keppeler et al. (2012) used $Q_{B}$ in conjunction with the model given by eq. 7 for the modelling of $\overline{\nabla \cdot(\rho D \nabla c)}+\overline{\dot{\omega}}$ for LES. The damping function $Q_{B}$ in the context of LES is given by (Bruneaux et al., 1997; Keppeler et al., 2012):

$$
Q_{B}=\exp [-2.0 \beta(\tilde{c}-\tilde{T})]
$$

The performances of the FSD closures given by eqs. 5 and 7 in predicting the filtered reactiondiffusion imbalance $\overline{\nabla \cdot(\rho D \nabla c)}+\overline{\dot{\omega}}$ in the near-wall region for head-on quenching of statistically planar turbulent premixed flames have been assessed in Section 4 of this paper.

\section{NUMERICAL IMPLEMENTATION}

A well-known three-dimensional compressible DNS code SENGA (Jenkins and Cant, 1999) has been used to simulate head-on quenching of statistically planar turbulent premixed flames. In SENGA the mass, momentum, energy and species conservation equations are solved in non- 
dimensional form. The spatial discretisation is carried out using a $10^{\text {th }}$ order central difference scheme for the internal grid points but the order of differentiation gradually decreases to a onesided $2^{\text {nd }}$ order scheme at the non-periodic boundaries. An explicit low-storage $3^{\text {rd }}$ order RungeKutta scheme is used for time advancement (Wray, 1990). The simulation domain is taken to be a rectangular box of size $70.6 \delta_{Z} \times 35.2 \delta_{Z} \times 35.2 \delta_{Z}$ (where the long-side of the domain is aligned with $x_{1}$-direction) which is discretised by a uniform Cartesian mesh of dimension $512 \times 256 \times 256$ ensuring at least 10 grid points across the thermal flame thickness $\delta_{t h}=$ $\left(T_{a d}-T_{0}\right) / \max |\nabla \hat{T}|_{L}$, where the sub-script $L$ denotes steady unstrained planar flame values. Furthermore, this resolution ensures that the normalised grid size $\rho_{0} u_{\tau} \Delta x / \mu_{0}$ remains smaller than unity, where $u_{\tau}$ and $\mu_{0}$ are the friction velocity and unburned gas viscosity, respectively. A no-slip isothermal inert wall with temperature $T_{w}=T_{0}$, with zero wall-normal mass flux is specified at $x_{1}=0$, and a partially non-reflecting outlet boundary condition is specified for the boundary opposite to the isothermal wall. The transverse directions are taken to be periodic. The non-periodic boundary conditions are specified using the Navier-Stokes Characteristic Boundary Conditions (NSCBC) technique (Poinsot and Lele, 1992). The wave amplitude variation entering into the partially-non-reflecting outflow boundary is taken to be $L=$ $s\left(1-M a_{m}^{2}\right) a\left(p-p_{\infty}\right) / L_{d}$ (where $s$ is the model parameter, $M a_{m}$ is the maximum Mach number, $a$ is the acoustic speed, $L_{d}$ is the domain length in the direction in question, $p$ is the instantaneous pressure and $p_{\infty}$ is the desired pressure), whereas linear relaxation expressions (i.e. $L=K\left(\varphi-\varphi^{t}\right)$ where $K$ is the relaxation parameter, $\varphi$ is the variable in question and $\varphi^{t}$ is the target value) in terms of target values of velocity components and temperature are specified for the incoming waves for partially non-reflecting inflow boundaries (Poinsot and Lele, 1992). Here $s=0.25$ is taken following the suggestiuon of Poinsot and Lele (1992). A Courant-Friedrichs-Lewy (CFL) number of about 0.1 (based on acoustic speed in the unburned gas) is used for these simulations which also satisfies the diffusive stability criterion for explicit 
time-marching. Furthermore, the timestep size for this CFL number remains smaller than the Kolmogorov timescale.

A steady unstrained planar laminar premixed flame solution is used to initialise the reactive field so that the $T=\left(\widehat{T}-T_{0}\right) /\left(T_{a d}-T_{0}\right)=0.9$ isosurface (the maximum value of chemical reaction rate is obtained close to $T=0.9$ in case of the laminar flame with present thermochemistry) remains at a distance $20 \delta_{Z}$ away from the wall. This allows for enough time for the flame to evolve before interacting with the wall. A standard pseudo-spectral method (Rogallo, 1981) has been used for initialising the turbulent velocity fluctuations away from the wall with the help of a homogeneous isotropic incompressible field. The velocity components are specified to be zero on the wall due to no-slip condition and the initial velocity field was allowed to evolve for one an initial eddy turn-over time (i.e. $t_{e}=l / u^{\prime}$ where $l$ is the integral length scale and $u^{\prime}$ is the root-mean-square (rms) turbulent velocity magnitude) before interacting with the flame. For the statistically planar cases considered in this work, the flame propagates towards the wall under decaying turbulence. The initial values of normalised rootmean-square (rms) turbulent velocity fluctuation $u^{\prime} / S_{L}$, the ratio of longitudinal integral length scale to thermal flame thickness $L_{11} / \delta_{t h}$ for the turbulent velocity field away from the wall are listed in Table 1 along with the corresponding values of Damköhler number $D a=L_{11} S_{L} / \delta_{t h} u^{\prime}$, Karlovitz number $K a=\left(u^{\prime} / S_{L}\right)^{3 / 2}\left(L_{11} / \delta_{t h}\right)^{-1 / 2}$ and turbulent Reynolds number based on $L_{11}$ (i.e. $\operatorname{Re}_{\mathrm{t}}=\rho_{0} u^{\prime} L_{11} / \mu_{0}$ where $\rho_{0}$ and $\mu_{0}$ are the unburned gas density and viscosity respectively). One will get an increase in the numerical value of $\operatorname{Re}_{t}$ by a factor of almost 2.35 if the integral length scale $l_{T}=\tilde{k}^{\frac{3}{2}} / \tilde{\epsilon}$ (where $\tilde{k}$ and $\tilde{\epsilon}$ are turbulent kinetic energy and its dissipation rate in the context of RANS respectively) is used. The turbulent Reynolds number values used here are comparable to the values used in the previous analyses (Boger $e t$ al., 1998; Charlette et al., 2002; Chakraborty and Klein, 2008; Katragadda et al., 2012a,b; 
Klein et al., 2016) which concentrated on the LES FSD closure in turbulent premixed flames. The simulations have been carried out until the wall heat flux becomes spatially uniform after flame quenching, which amounts to either greater than or equal to $12 \delta_{Z} / S_{L}$ (where $12 \delta_{Z} / S_{L}$ corresponds to 21, 30, 21, 15 and 21 initial eddy turn over times for cases A-E respectively) for the cases considered here. Although flame-turbulence interaction takes place under decaying turbulence in this configuration, the turbulence intensity remains significant at the time instants for which the results are presented in this analysis. Interested readers are referred to Lai et al. (2017) for further information on spatial distribution of turbulent velocity fluctuation at different time instants (see Fig. 2 in Lai et al. (2017)). It is worth noting that several DNS studies have been carried out under decaying turbulence in the past (Boger et al., 1998; Charlette et al., 2002; Chakraborty and Klein, 2008; Katragadda et al., 2012a,b; Klein et al., 2016), which concentrated on LES closures of Flame Surface Density (FSD). The same approach has been adopted here. The findings based on decaying turbulence DNS studies in the context of FSD closure have been found to remain valid also for actual LES simulations of flames in experimental configurations (e.g. Ma et al., 2013, 2014a; Allauddin et al., 2017).

Standard values are considered for the Zel'dovich number $\beta$ and the ratio of specific heats $\gamma$ (i.e. $\beta=6.0$ and $\gamma=1.4$ ), $T_{a c}$ is the activation temperature. The heat release parameter $\tau$ and the global Lewis number (i.e. ratio of thermal diffusivity to mass diffusivity) are taken to be 6.0 and 1.0 for all cases considered here. To date, almost all FSD based closures have been proposed for unity Lewis number flames (Boger et al., 1998; Butz et al., 2015; Chakraborty and Cant, 2007, 2009; Chakraborty and Klein, 2008; Fureby, 2005; Hawkes and Cant, 2000, 2001; Hernandez-Perez et al., 2011; Ma et al., 2013, 2014a,b; Keppeler et al., 2014; Klein et al., 2016; Reddy and Abraham, 2012) and thus the global Lewis number is considered to be unity. The values of $\tau=6.0$ and $\beta=6.0$ are representative of iso-octane-air mixture with 
unburned gas temperature $T_{0}=325 \mathrm{~K}$ and equivalence ratio of 1.10 under atmospheric pressure.

The DNS data has been explicitly LES filtered using a Gaussian filter kernel $\boldsymbol{G}(\overrightarrow{\boldsymbol{r}})$ so that the LES filtered values of a general quantity $\boldsymbol{Q}$ can be calculated as follows for the internal grid points:

$$
\overline{Q(\vec{x})}=\int Q(\vec{x}-\vec{r}) G(\vec{r}) d \vec{r}
$$

The three-dimensional filter kernel is expressed as the tensor product of three one-dimensional filters:

$$
G(x, y, z)=G(x) \cdot G(y) \cdot G(z) \quad \text { with } \quad G(x)=\left(6 / \pi \Delta^{2}\right)^{1 / 2} \exp \left(-6 x^{2} / \Delta^{2}\right)
$$

This allows for a very efficient implementation of the filtering operation following Kempf et al. (2012). The filtering is performed in parallel using domain decomposition where each local domain has to be surrounded by a buffer region of the dimension of the order of LES filter width $\Delta$ such that the filter kernel approaches approximately zero when the convolution operation is performed for points close to the border of the local (decomposed) domain. For the grid points either at the wall or in the vicinity of the wall, a truncated version of $G(x, y, z)$ is used, and the filtered value for the near-wall points for the truncated filter is expressed as: $\overline{Q(\vec{x})}=\int Q(\vec{x}-\vec{r}) G(\vec{r}) d \vec{r} / \int G(\vec{r}) d \vec{r}$. It is worth noting that $\int G(\vec{r}) d \vec{r}$ is exactly equal to 1.0 for the internal grid points but the integral $\int G(\vec{r}) d \vec{r}$ yields non-unity value for truncated filters.

It is ensured $\partial \bar{c} / \partial x_{1}=0, u_{\Delta}^{\prime}=0, \overline{\hat{T}}=T_{0}$ and $\widetilde{\hat{T}}=T_{0}$ are maintained at the wall (i.e. $x_{1}=0$ ). In this paper results will be presented for filter sizes ranging from $\Delta \approx 0.4 \delta_{t h}$ where the flame is almost resolved, up to $\Delta \approx 2.8 \delta_{t h}$ where the flame becomes fully unresolved and $\Delta$ is 
comparable to the integral length scale $l$. Explicit filtering provides a dataset with the same dimensions as the original DNS database for each LES filter width $\Delta$. For the purpose of $a$ priori analysis one has to decide if the gradient of a variable under consideration in a model expression is evaluated based on the DNS grid size $\Delta x_{D N S}$ or based on the size of the convolution filter $\Delta x_{\text {conv }}$ which corresponds $\Delta x_{c o n v}=n \Delta x_{D N S}$ with $n=4,8,12,16,20,24,28$ for this analysis. According to Liu et al. (1994) a-priori assessment of model expressions should be based solely on variables sampled on the coarse grid. However, one obtains the combined effects of truncation errors due to finite difference formulas and modelling errors in this approach. By contrast, evaluating the gradients on DNS grid using the same numerical methodology followed in DNS eliminates the effects of truncation errors and shows the true potential of the model without any adulteration due to numerical issues. A $2^{\text {nd }}$ order finitedifference scheme is used for LES grid based model evaluations in this analysis. Both approaches will be adopted for the analysis shown in Section 4.

\section{RESULTS \& DISCUSSION}

The presentation of the results precedes in several steps. After a short explanation of the flame physics in section 4.1, the modelling of generalised FSD is discussed together with the FSD damping factor $Q_{A}$ as well as the newly suggested expressions given in Eqs. 12 and 13. The spatial distribution of FSD is analysed in section 4.2, whereas the local model behaviour is discussed in section 4.3. In order to close the filtered reaction diffusion imbalance term, besides FSD, a closure for surface averaged density weighted displacement speed is required. The modelling of $\overline{\dot{\omega}+\nabla \cdot(\rho D \nabla c)}$ is covered in sections 4.4 (spatial distribution) and 4.5 (local behaviour) together with the consumption rate damping factor $Q_{B}$ and the new model given in Eq. 14. 


\subsection{Global description of flame quenching in terms of wall heat flux and Peclet number}

The instantaneous distributions of reaction progress variable $c$ and non-dimensional temperature $T$ at different time instants for case $E$ are shown in Fig. 1. It can be seen from Fig. 1 that the distributions of $c$ and $T$ resemble each other when the flame is away from the wall but these distributions become significantly different from each other once the quenching is initiated. It is worth noting that $c=T$ for low Mach number, globally adiabatic, unity Lewis number flames but this equality does not hold in head-on quenching due to loss of adibaticity. Furthermore, the wall boundary conditions for $c$ and $T$ are different at the wall (i.e. Neumann and Dirichlet boundary conditions respectively), which also contributes to the inequality between $c$ and $T$ (i.e. $c \neq T$ ). The flame-wall interaction in head-on quenching is often characterised by a wall Peclet number $P e=X / \delta_{Z}$ and the normalised wall heat flux magnitude $\Phi=\left|q_{w}\right| /\left[\rho_{0} S_{L} C_{P}\left(T_{a d}-T_{0}\right)\right]$ where $X$ is the wall normal distance of $T=0.9$ isosurface and $q_{w}=-\lambda\left(\partial \hat{T} / \partial x_{1}\right)_{w}$ is the instantaneous wall heat flux with $\lambda$ being the thermal conductivity. The temporal evolutions of the maximum, minimum and mean values of $P e$ and $\Phi$ for case $\mathrm{E}$ are also shown in Fig. 1 along with the corresponding variation obtained for head-on quenching of a laminar one-dimensional premixed flame. Figure 1 demonstrates that a decrease in $P e$ in the laminar flame due to flame propagation towards the cold wall leads to an increase in wall heat flux magnitude $\Phi$ with time. The minimum Peclet number is obtained when the flame quenches (i.e. when the $T=0.9$ isosurface is the closest to the wall), and this time is almost coincident with the time instant at which the maximum value of the normalised wall heat flux magnitude $\Phi$ is obtained (see Fig. 1). The isotherms move away from the cold wall following the flame quenching, which gives rise to a continuous decrease (increase) in $\Phi(P e)$ with time. For the laminar flame calculation, the minimum value of Peclet number is found to be $\left(P e_{\min }\right)_{L}=2.83$, whereas the maximum normalised heat flux magnitude is obtained as $\left(\Phi_{\max }\right)_{\mathrm{L}}=0.34$. These values are in agreement with previous experimental (Huang et al., 
1986; Jarosinsky, 1986; Vosen et al., 1984) and computational (Poinsot et al., 1993) findings. Figure 1 shows that the temporal variations of $P e$ and $\Phi$ in turbulent flames remain qualitatively similar to the corresponding variations in the laminar premixed flame. However, $\Phi_{\max }$ in the turbulent case is greater than the corresponding value in the one-dimensional laminar flame. Turbulent velocity fluctuation induce flame wrinkling and generate flame surface area, which in turn increases the overall burning rate. The increases in burning rate and flame area generation are reflected in the higher value of $\Phi_{\max }$ in turbulent flames than the corresponding laminar flame value (i.e. $\left.\left(\Phi_{\max }\right)_{\mathrm{L}}\right)$. Flame wrinkling in turbulent flames gives rise to a separation between the maximum and minimum values of $P e$ and $\Phi$. Furthermore, Fig. 1 shows that the minimum value of wall Peclet number $P e_{\min }$ in the turbulent flame remains close to the corresponding value in the case of a laminar one-dimensional premixed flame-wall interaction. The temporal evolutions of $P e$ and $\Phi$ for other turbulent cases are qualitatively similar to those in case E, and thus are not explicitly shown here. Interested readers are directed to Lai and Chakraborty (2016a,b) for further discussion on temporal evolutions of $P e$ and $\Phi$ in turbulent cases A-E. The temporal evolutions of the normalised turbulent flame speed $S_{T} / S_{L}$ (where $S_{T}=\left(\rho_{0} A_{p}\right)^{-1} \int_{V} \dot{W} d V$, and $A_{p}$ is the projected area in the direction of flame propagation), and normalised flame surface area $A_{T} / A_{L}$ (where flame surface area has been evaluated here using the volume integral $A=\int_{V}|\nabla c| d V$, and turbulent and laminar values are shown with subscripts $\mathrm{T}$ and $\mathrm{L}$ respectively) values are reported in Table 2, which also shows that the rate of flame propagation is faster for higher values of $u^{\prime} / S_{L} \sim R e_{t}^{1 / 4} \mathrm{Ka}^{1 / 2} \sim R e_{t}^{1 / 2} D a^{-1 / 2}$ when the flame is away from the wall and flame quenching initiates at an earlier time instant for higher values of $u^{\prime} / S_{L}$. As the extent of flame wrinkling increases with increasing $u^{\prime} / S_{L}$, the flame wrinkles reach close to the wall at an earlier time for higher values of $u^{\prime} / S_{L}$ (Lai and Chakraborty, 2016a-c). Thus, the cases with high values of 
$u^{\prime} / S_{L}$ exhibit smaller values of $A_{T} / A_{L}$ and $S_{T} / S_{L}$ than the cases with small values of $u^{\prime} / S_{L}$ at later times (e.g. $t \geq 6 \delta_{Z} / S_{L}$ ), because by then the flame is in more advanced stage of quenching in these cases than in the cases with small $u^{\prime} / S_{L}$ (e.g. case $\mathrm{E}$ (case $\mathrm{C}$ ) is at more advanced stage of flame quenching than case $\mathrm{C}$ (case A)).

\subsection{Spatial distribution of generalised FSD and its model predictions}

The variations of normalised FSD averaged over the homogeneous direction (i.e. $\left\langle\Sigma_{g e n}\right\rangle \times$ $\delta_{Z}=\int \Sigma_{g e n} d y d z / L_{y} L_{z} \times \delta_{Z}$ where $L_{y}$ and $L_{z}$ are the domain lengths in y and z directions) in the normalised wall direction $x_{1} / \delta_{Z}$, as obtained from DNS data and from FUREBY and KEPPELER models, at different time instants and filter widths are shown in Figs. 2 and 3. ${ }^{1}$ Only cases A, C and E are shown in Figs. 2 and 3 and in subsequent figures because of qualitative similarity between cases $\mathrm{A}$ and $\mathrm{B}$ and between $\mathrm{D}$ and $\mathrm{E}$.

For the evaluation of model predictions, $|\nabla \bar{c}|$ is calculated using finite-difference formulae on the equivalent convolution filter width $\Delta \mathrm{x}_{\text {conv }}$ for Fig. 3, whereas the spatial differentiation is done using the DNS grid $\Delta x_{D N S}$ for Fig. 2. In some sense the results in Fig. 2 can be understood as an assessment of the model expression itself, whereas in Fig. 3 the disagreement between the model predictions with DNS data arises due to combined actions of modelling and unavoidable numerical errors. Thus, the difference in model predictions between Figs. 2 and 3 originates due to numerical errors and its interaction with modelling errors. The resolved part of FSD (i.e. $|\nabla \bar{c}|$ ) plays an important role to the total contribution $\Sigma_{g e n}$ with decreasing filter width $\Delta\left(\because \lim _{\Delta \rightarrow 0} \Sigma_{g e n}=\lim _{\Delta \rightarrow 0}|\nabla \bar{c}|=|\nabla c|\right)$, and thus the model does not play a significant role in the limit of small filter size. Hence, the predictions of the models are in good agreement

\footnotetext{
${ }^{1}$ Equations 12 and 13 are introduced later and their predictions will be discussed in detail in this sub-section.
} 
with $\left\langle\Sigma_{g e n}\right\rangle$ extracted from DNS data for $\Delta \leq \delta_{t h}$. However, the predictions of FUREBY and KEPPELER models are significantly different for $\Delta \gg \delta_{t h}$. It can be seen from Figs. 2 and 3 that the FUREBY model prediction agrees with DNS data for all cases for all filter widths. However, the FUREBY model underpredicts $\left\langle\Sigma_{g e n}\right\rangle \times \delta_{Z}$ slightly in the region close to the wall (i.e. $2 \leq x_{1} / \delta_{Z} \leq 3$ ) and this behaviour is prominent for $\Delta>\delta_{t h}$ and the extent of this underprediction is relatively higher for the case, where the numerical differentiation is done using $\Delta x_{c o n v}$, than the values obtained due to differentiation using $\Delta x_{D N S}$. By contrast, the KEPPELER model significantly overpredicts $\left\langle\Sigma_{g e n}\right\rangle \times \delta_{Z}$ obtained from DNS data for all cases for $\Delta>\delta_{t h}$. The extent of overprediction of KEPPELER model is relatively smaller for the model prediction based on the finite-difference formulae using the equivalent LES grid size $\Delta x_{c o n v}$ than in the case where $\Delta x_{D N S}$ is used for model evaluation. This can be explained by the fact that finite difference gradients on coarse grids underestimate the real gradient as can be seen from the modified wavenumber diagram (Klein et al., 2016).

As the near-wall damping proposed by Alshaalan and Rutland (1998) was originally applied to the FSD, the predictions of $\left\langle\mathrm{Q}_{\mathrm{A}} \Sigma_{\text {gen }}\right\rangle \times \delta_{Z}$ according to both FUREBY and KEPPELER models, at different time instants and filter widths are shown in Figs. 2 and 3. It can be seen that the predictions of $\left\langle\mathrm{Q}_{\mathrm{A}} \Sigma_{g e n}\right\rangle \times \delta_{Z}$ according to both FUREBY and KEPPELER models significantly underpredict the magnitude of $\left\langle\Sigma_{g e n}\right\rangle \times \delta_{Z}$ extracted from DNS data.

It is worth noting that $\int\left\langle\Sigma_{g e n}\right\rangle d x=\int \Sigma_{g e n} d x d y d z / L_{y} L_{z}$ and thus a good agreement of the model prediction with the distribution of $\left\langle\Sigma_{g e n}\right\rangle \times \delta_{Z}$ with $x_{1} / \delta_{Z}$ obtained from DNS suggests that the model is capable of predicting the volume-integrated FSD (i.e. $\int \Sigma_{g e n} d x d y d z$ ) which represents total flame surface area. The findings based on Figs. 2 and 3 suggest that the FUREBY model is more successful in predicting the total flame surface area than the 
KEPPELER model and the variants of the FUREBY and KEPPELER models with the damping factor $Q_{A}$. Furthermore, a comparison between Figs. 2 and 3 reveals that the FUREBY model evaluated on DNS grid is more successful in predicting the total flame surface area than the variant of the same model evaluated using the equivalent LES grid size.

As the performance of the FUREBY model has been found to be more promising than the KEPPELER model based on the observations made from Figs. 2 and 3, the near-wall modification has been proposed here for the FUREBY model. It is worth noting that $u_{\Delta}^{\prime}$ assumes zero at the wall and thus the FUREBY model yields $\Sigma_{g e n}=|\nabla \bar{c}|$ at the wall. The observations from Figs. 2 and 3 indicate that the original version of the FUREBY model underpredicts the magnitude of $\Sigma_{g e n}$ close to the wall. Both figures show that this is to a large extent an effect of filtering the data as the underprediction is more pronounced for large filter widths (i.e. $\Delta / \delta_{t h} \gg 1$ ). Thus the correction factor needs to be such that it assumes a value greater than unity close to the wall but it asymptotically approaches unity away from the wall (i.e. $\left.x_{1} / \delta_{Z} \gg P e_{\min }\right)$. Here, the following near-wall modification has been proposed in the following manner for the FUREBY model when DNS grid spacing is used for the evaluation of $\Sigma_{g e n}$ :

$$
\Sigma_{g e n}=\exp \left[-0.25 E_{1}\left(1-f_{b}\right)\right]\left(1+\Gamma \cdot u_{\Delta}^{\prime} / S_{L}\right)^{D_{f}-2}|\nabla \bar{c}|
$$

where $\left(1+\Gamma \cdot u_{\Delta}^{\prime} / S_{L}\right)^{D_{f}-2}|\nabla \bar{c}|$ is taken from the FUREBY model expression and the model parameter $E_{1}=0.5\left[\operatorname{erf}\left(x_{1} / \delta_{Z}-P e_{\min }\right)-1\right]$ accounts for near-wall behaviour for large filter widths (i.e. $\left.\Delta \gg \delta_{t h}\right)$. This requirement is met by $\exp \left[-0.25 E_{1}\left(1-f_{b}\right)\right]$ because $E_{1}=$ $0.5\left[\operatorname{erf}\left(x_{1} / \delta_{Z}-P e_{\min }\right)-1\right]$ assumes negative value close to the wall and $\left(1-f_{b}\right)=\{1-$ $\left.\exp \left[-0.5\left(\Delta / \delta_{t h}\right)^{1.7}\right]\right\}$ approaches unity for $\Delta / \delta_{t h} \gg 1$ where the flame remains completely unresolved However, for $x_{1} / \delta_{Z} \gg P e_{\min }$, the term $\operatorname{erf}\left(x_{1} / \delta_{Z}-P e_{\min }\right)$ becomes unity and 
thus $E_{1}$ and $\exp \left[-0.25 E_{1}\left(1-f_{b}\right)\right]$ asymptotically vanish and approach unity, respectively. For small values of $\Delta$ (e.g. $\Delta \rightarrow 0)$ the term $\left(1-f_{b}\right)$ goes to zero and thus the correction factor $\exp \left[-0.25 E_{1}\left(1-f_{b}\right)\right]$ becomes unity. As discussed below, Eq. 12 gives satisfactory results when finite differences are calculated on the DNS grid as in Fig. 2. However, due to the difference between the model prediction evaluations based on DNS and LES grid spacings, an additional correction is needed when the FUREBY model is modified for near-wall prediction, which is given by:

$$
\Sigma_{g e n}=\exp \left[-0.25 E_{1} G_{x}\left(1-f_{b}\right)\right]\left(1+\Gamma \cdot u_{\Delta}^{\prime} / S_{L}\right)^{D_{f}-2}|\nabla \bar{c}|
$$

where

$$
G_{x}=A_{w} \exp \left[-0.5\left(\frac{x_{1} / \delta_{Z}-P e_{\min }}{1.2}\right)^{2}\right]
$$

and $A_{w}=3.0(\tilde{c}-\tilde{T})^{0.4}$ is a model parameter which takes effect due to non-adibaticity close to the wall where $\tilde{c} \neq \tilde{T}$ (see Fig. 1), and the same approach was used by Alshalaan and Rutland (1998) and Bruneaux et al. (1997). The variation of the FUREBY model prediction in the near-wall region when $|\nabla \bar{c}|$ is evaluated using fine-difference formulae is found to be qualitatively different from the predictions based on DNS grid size but it continues to underpredict $\Sigma_{g e n}$ close to the wall for large filter widths (i.e. $\Delta / \delta_{t h} \gg 1$ ). Thus, the correction factor needs to be modified for the FSD evaluation when the derivatives are evaluated over the equivalent LES grid and this is accounted for by a correction factor given by $\exp \left[-0.25 E_{1}{ }^{\prime}\left(1-f_{b}\right)\right]$ where $E_{1}^{\prime}=G_{x} E_{1}$ where the limiting behaviours of $\exp \left[-0.25 E_{1}{ }^{\prime}(1-\right.$ $\left.f_{b}\right)$ ] in eq. 13 with respect to filter width and wall normal distance are qualitatively similar to the aforementioned behaviours of $\exp \left[-0.25 E_{1}\left(1-f_{b}\right)\right]$ in the context of eq. 12 .

A comparison between Figs. 2 and 3 reveals that the FUREBY model evaluated on LES grid underpredicts $\left\langle\Sigma_{g e n}\right\rangle \times \delta_{Z}$ for $0<x_{1} / \delta_{Z}<P e_{\min }$ due to truncation error on top of 
underprediction resulting from the modelling error. The model parameter $G_{x}$ compensates for the observed underprediction of FSD in the region around $x_{1} / \delta_{Z} \approx P e_{\min }$ when the FUREBY model is evaluated on LES grid. It is admitted that a degree of empiricism is involved in the correction factors used in eqs. 12 and 13, but the extent of this empiricism is comparable to that involved in the correction factors (i.e. $Q_{A}$ ) proposed by Alshaalan et al. (1998) for the nearwall FSD based closures. Moreover, these correction factors are taken to be functions of the minimum Peclet number $P e_{\min }$ (which quantifies the quenching distance), normalised wall normal distance $x_{1} / \delta_{Z}$ and $(\tilde{c}-\tilde{T})$ (which accounts for enthalpy loss in the vicinity of the wall (Bruneaux et al., 1996)), which were not considered in the correction factor $Q_{A}$ proposed earlier by Alshaalan et al. (1998).

\subsection{Local behaviour of the FSD models}

Ideally, an algebraic model for the generalised FSD should predict the total flame surface area (which is given by the volume integral of the model prediction) accurately for all filter widths. Moreover, the models need to accurately capture both quantitative and qualitative behaviours of FSD obtained from DNS data. Finally, in the context of LES, the model needs to capture the correct local behaviour which can be quantified by the correlation coefficient between the model prediction and the generalised FSD extracted from DNS data. These criteria were discussed elsewhere (Chakraborty and Klein, 2008; Katragadda et al., 2012a,b; Klein et al., 2016) in detail. Figures 2 and 3 show if the models predict the correct magnitude of the generalised FSD and a good agreement with the model prediction with the distribution of $\left\langle\Sigma_{g e n}\right\rangle \times \delta_{Z}$ with $x_{1} / \delta_{Z}$ obtained from DNS suggests that the model is capable of predicting the volume-integrated FSD (i.e. $\int \Sigma_{g e n} d x d y d z$ ) which represents total flame surface area because $\int\left\langle\Sigma_{g e n}\right\rangle d x$ is given by $\int\left\langle\Sigma_{g e n}\right\rangle d x=\int \Sigma_{g e n} d x d y d z / L_{y} L_{z}$. However, the results shown in Figs. 2 and 3 do not ensure that the models capture the local behaviour of the 
generalised FSD adequately. In order to predict the local behaviour of the FSD the correlation coefficient between the FSD extracted from DNS and the model prediction should be as close to unity as possible (Chakraborty and Klein, 2008; Katragadda et al., 2012a,b; Klein et al., 2016).The correlation coefficients between $\Sigma_{g e n}$ and the model predictions using the samples $0.01 \leq \bar{c} \leq 0.99$ for different filter widths at different time instants are shown in Fig. 6 for both model evaluations using DNS and equivalent LES grid spacing. The samples corresponding to $\bar{c}<0.01$ and $\bar{c}>0.99$ are ignored, because both $\Sigma_{g e n}$ and the model predictions assume small values for these samples, and thus the correlation coefficient values for both $\bar{c}<0.01$ and $\bar{c}>0.99$ are unlikely to yield any meaningful information. It can be seen from Fig. 4a that the FUREBY model shows high positive correlation for all filter widths for all cases considered here when the flame is away from the wall. The correlation coefficient between $\Sigma_{g e n}$ extracted from DNS data and the KEPPELER model evaluated on DNS grid remains comparable to (but mostly smaller than) that in the case of the FUREBY model for all filter widths when the flame is away from the wall. However, the correlation coefficients of both the FUREBY and KEPPELER models drop significantly as the quenching progresses (see $t_{5}=10 \delta_{Z} / S_{L}$ in cases $\mathrm{C}$ and $\left.\mathrm{E}\right)$. The introduction of the damping factor $Q_{A}$ further reduces the correlation coefficient for both the FUREBY and KEPPELER models.

The KEPPELER model exhibits higher correlation coefficient than the FUREBY model for $\Delta>\delta_{t h}$ for case A at all times shown in Fig. $4 \mathrm{~b}$ when the models are evaluated on the equivalent LES grid. A qualitatively similar trend has been observed until $t<6 \delta_{Z} / S_{L}$ for all filter widths in cases $\mathrm{C}$ and $\mathrm{E}$. By contrast, the FUREBY model shows either almost the same or slightly greater correlation in comparison to the KEPPELER model for $\Delta=2.8 \delta_{t h}$ when the models are evaluated on the equivalent LES grid. The presence of $Q_{A}$ reduces the correlation coefficient for both the FUREBY and KEPPELER models. A comparison between Figs. 4a 
and $4 \mathrm{~b}$ reveals that the correlation coefficients between $\Sigma_{g e n}$ extracted from DNS data with model predictions are higher when the models are evaluated on DNS grid than the predictions based on the equivalent LES grid spacing. The flame wrinkles for cases with higher $u^{\prime} / S_{L}$ reach the wall earlier and as a result of this cases $\mathrm{C}$ and $\mathrm{E}$ show a more advanced stage of quenching at $t_{5}=10 \delta_{Z} / S_{L}$ than in case A (see Lai and Chakraborty (2016a,b) for further discussion on this).

It can be seen from Figs. $4 \mathrm{a}$ and $4 \mathrm{~b}$ that the modified model expressions eq. 12 and eq. 13 yield correlation coefficients which are comparable to the FUREBY model. Furthermore, Figs. 6a and $6 \mathrm{~b}$ show that eq. 12 and eq. 13 exhibit the highest correlation coefficients during flamewall interaction amongst all the model expressions considered here (e.g. $t_{5}$ for cases $\mathrm{C}$ and $\mathrm{E}$ ).

\subsection{Prediction of the spatial distribution of the reaction-diffusion imbalance term $\overline{\dot{\omega}+\nabla \cdot(\rho D \nabla c)}$}

The comparison between $\left\langle\overline{\left(\rho S_{d}\right)_{S}} \Sigma_{g e n}\right\rangle\left(\left\langle Q_{A} \overline{\left(\rho S_{d}\right)}{ }_{s} \Sigma_{g e n}\right\rangle\right)$ and $\langle\overline{\dot{\omega}+\nabla \cdot(\rho D \nabla c)}\rangle$ when $\overline{\left(\rho S_{d}\right)_{S}}$ is extracted from DNS data and $\Sigma_{g e n}\left(Q_{A} \Sigma_{g e n}\right)$ evaluated according to the FUREBY and KEPPELER models remains qualitatively similar to that between the respective model predictions with FSD extracted from DNS data (as shown earlier in Figs. 2 and 3). Thus the comparison between $\left\langle{\overline{\left(\rho S_{d}\right)}}_{s} \Sigma_{g e n}\right\rangle\left(\left\langle Q_{A}{\overline{\left(\rho S_{d}\right)}}_{s} \Sigma_{g e n}\right\rangle\right)$ and $\langle\overline{\dot{\omega}+\nabla \cdot(\rho D \nabla c)}\rangle$ is not explicitly shown here. There have been several previous a-priori DNS analyses (Boger et al., 1998; Klein et al., 2016) and LES studies (Butz et al., 2015; Hawkes and Cant, 2000,2001; HernandezPerez et al., 2011; Ma et al., 2013,2014b) where ${\overline{\left(\rho S_{d}\right)}}_{s}$ was approximated as: $\overline{\left(\rho S_{d}\right)_{s}}=\rho_{0} S_{L}$. The variations of $\langle\overline{\dot{\omega}+\nabla \cdot(\rho D \nabla c)}\rangle \times \delta_{Z} / \rho_{0} S_{L}$ and $\left\langle\rho_{0} S_{L} \Sigma_{g e n}\right\rangle \times \delta_{Z} / \rho_{0} S_{L}$ in the normalised wall direction $x_{1} / \delta_{Z}$, as obtained from DNS data, are shown in Figs. 5 and 6 for different time instants and filter widths along with the model predictions of $\left\langle\rho_{0} S_{L} \Sigma_{g e n}\right\rangle \times \delta_{Z} / \rho_{0} S_{L}$ evaluated 
on DNS and LES grid spacings, respectively. ${ }^{2}$ It is worth noting that the results shown in Fig. 5 provide the true potential of the proposed reaction-diffusion imbalance and any disagreement between DNS data and model prediction originates due to modelling inaccuracy. By contrast, the results shown in Fig. 6 exhibit the combined action of modelling and numerical errors and thus the disagreement between DNS data and model predictions originate due to both modelling and numerical inaccuracies. Figures 5-6 reveal that $\left\langle\rho_{0} S_{L} \Sigma_{\text {gen }}\right\rangle$ and conditional mean value of $\rho_{0} S_{L} \Sigma_{g e n}$, as obtained from DNS data, capture the variations of $\langle\overline{\dot{\omega}+\nabla \cdot(\rho D \nabla c)}\rangle$ and conditional mean value of $\overline{\dot{\omega}+\nabla \cdot(\rho D \nabla c)}$ respectively for all filter widths when the flame remains away from the wall. However, $\left\langle\rho_{0} S_{L} \Sigma_{\text {gen }}\right\rangle$ tends to significantly overpredict $\langle\overline{\dot{\omega}+\nabla \cdot(\rho D \nabla c)}\rangle$ in the near-wall region during flame quenching. The term $\left\langle\rho_{0} S_{L} \Sigma_{g e n}\right\rangle \times$ $\delta_{Z} / \rho_{0} S_{L}$ according to the FUREBY and KEPPELER models overpredicts $\langle\overline{\dot{\omega}+\nabla \cdot(\rho D \nabla c)}\rangle \times$ $\delta_{Z} / \rho_{0} S_{L}$ during advanced stages of flame quenching (e.g. $t_{5}=10 \delta_{Z} / S_{L}$ in cases C and E) in the vicinity of the wall for both DNS and LES grid based evaluations. However, $\left\langle\rho_{0} S_{L} \Sigma_{g e n}\right\rangle$ according to the FUREBY model evaluation using $\Delta x_{D N S}$ satisfactorily captures the behaviour of $\langle\overline{\dot{\omega}+\nabla \cdot(\rho D \nabla c)}\rangle$ when the flame is away from the wall, and a similar qualitative behaviour is observed for the conditional mean values. By contrast, $\left\langle\rho_{0} S_{L} \Sigma_{g e n}\right\rangle$ according to the FUREBY model evaluation using $\Delta x_{\text {conv }}$ slightly underpredicts $\langle\overline{\dot{\omega}+\nabla \cdot(\rho D \nabla c)}\rangle$ when the flame is away from the wall. The KEPPELER model (for both DNS and LES grid based evaluations) leads to an overprediction of $\langle\overline{\dot{\omega}+\nabla \cdot(\rho D \nabla c)}\rangle$ by $\left\langle\rho_{0} S_{L} \Sigma_{g e n}\right\rangle$ away from the wall, and a qualitatively similar behaviour has been observed for the variations of the mean values of the corresponding quantities conditional on $\bar{c}$. The quantity $\left\langle Q_{A} \rho_{0} S_{L} \Sigma_{\text {gen }}\right\rangle$ does not adequately capture the

\footnotetext{
${ }^{2}$ Equation 14 is introduced later and its prediction will be discussed in detail in this sub-section.
} 
qualitative and quantitative variations of $\langle\overline{\dot{\omega}+\nabla \cdot(\rho D \nabla c)}\rangle$ both away from and near to the wall for both FUREBY and KEPPELER models for DNS and LES grid based model evaluations.

Bruneaux et al. (1997) proposed a damping factor $Q_{B}$ for $\rho_{0} S_{L}$ to account for reduced burning rate due to flame quenching in the near-wall region. Figures 5 and 6 demonstrate that $\left\langle Q_{B} \rho_{0} S_{L} \Sigma_{\text {gen }}\right\rangle$ remains in good agreement with $\left\langle\rho_{0} S_{L} \Sigma_{\text {gen }}\right\rangle$ away from the wall for both FUREBY and KEPPELER models but $Q_{B}$ severely damps the magnitude of the reactiondiffusion imbalance close to the wall and yields a negligible value of $\left\langle Q_{B} \rho_{0} S_{L} \Sigma_{\text {gen }}\right\rangle$ close to the wall even when $\langle\overline{\dot{\omega}+\nabla \cdot(\rho D \nabla c)}\rangle$ assumes significant values. Thus, $Q_{B} \rho_{0} S_{L} \Sigma_{g e n}$ cannot be considered as a viable model for $\overline{\dot{\omega}+\nabla \cdot(\rho D \nabla c)}$ in the near-wall region for the purpose of LES of head-on quenching.

Based on the observations made from Figs. 5-6, a near-wall modification for the filtered reaction-diffusion imbalance has been proposed here in the following manner where $\Sigma_{g e n}$ is evaluated using eq. 12 (eq. 13) for DNS (LES) grid spacing:

$$
\overline{\dot{\omega}+\nabla \cdot(\rho D \nabla c)}=Q_{J} \rho_{0} S_{L} \Sigma_{g e n} \text { where } Q_{J}=\exp \left[0.9 E_{2}\left(x_{1} / \delta_{Z}\right)^{2}\right]^{\tilde{c}-\tilde{T}}
$$

where the parameter $E_{2}=0.5\left[\operatorname{erf}\left(x_{1} / \delta_{Z}-2 P e_{\min }\right)-1\right]$ ensures that $Q_{J} \rho_{0} S_{L}$ approaches $\rho_{0} S_{L}$ away from the wall (i.e. $x_{1} / \delta_{Z} \gg P e_{\text {min }}$ ), whereas $Q_{j}$ is primarily responsible for damping the consumption rate once the flame quenching is initiated (i.e. $\tilde{c} \neq \tilde{T}$ ) when $\tilde{c}$ tends to assume greater value than $\widetilde{T}$. Admittedly a degree of empiricism is involved in the correction factor $Q_{J}$ used in eq. 14 but the extent of this empiricism is comparable to that involved in the correction factor (i.e. $Q_{B}$ ) proposed earlier by Bruneaux et al. (1996) for the near-wall reaction rate closure. Moreover, the correction factor $Q_{J}$ is a function of the minimum Peclet number $P e_{\min }$ (which quantifies the quenching distance), normalised wall normal distance $x_{1} / \delta_{Z}$ and 
$(\tilde{c}-\tilde{T})$ (which accounts for enthalpy loss in the vicinity of the wall). For the convenience of the readers the full model expression for the filtered reaction diffusion imbalance term, to be used in a LES simulation is recalled

$\overline{\dot{\omega}+\nabla \cdot(\rho D \nabla c)}=Q_{J} \rho_{0} S_{L} \exp \left[-0.25 E_{1} G_{x}\left(1-f_{b}\right)\right]\left(1+\Gamma \cdot u_{\Delta}^{\prime} / S_{L}\right)^{D_{f}-2}|\nabla \bar{c}|$

where $G_{x}$ and $Q_{J}$ are given by eqs. 13 ii and 14 respectively. It can be seen from Figs. 5-6 that the prediction of eq. 14 remains better than the other alternatives available in existing literature.

\subsection{Local behaviour of the reaction-diffusion imbalance term}

Figures 5 and 6 exhibit if the closures for the reaction-diffusion imbalance term capture the spatial distribution and the magnitude of $\langle\overline{\dot{\omega}+\nabla \cdot(\rho D \nabla c)}\rangle$ extracted from DNS accurately. However, a good agreement between the model prediction and $\langle\overline{\dot{\omega}+\nabla \cdot(\rho D \nabla c)}\rangle$ obtained from DNS is not sufficient in the context of LES, and an ideal model needs to accurately capture the local behaviour of $\overline{\dot{\omega}+\nabla \cdot(\rho D \nabla c)}$. The correlation coefficient between the model prediction and $\overline{\dot{\omega}+\nabla \cdot(\rho D \nabla c)}$ needs to be as close to unity as possible so that the model captures the local behaviour of the reaction-diffusion term obtained from DNS data. The correlation coefficients between $\overline{\dot{\omega}+\nabla \cdot(\rho D \nabla c)}$ and the model predictions using the samples $0.01 \leq \bar{c} \leq 0.99$ for different filter widths at different time instants are shown in Fig. 7 for model evaluations using finite-difference formulae using the equivalent LES grid spacing. It is evident from Fig. 7 that

the correlation coefficient between $\overline{\dot{\omega}+\nabla \cdot(\rho D \nabla c)}$ extracted from DNS data and $\overline{\left(\rho S_{d}\right)_{s}} \Sigma_{g e n}$ according to the KEPPELER model evaluated on the DNS grid spacing remains comparable to (but mostly smaller than) that in the case of the FUREBY model for all filter widths when the flame is away from the wall. However, the correlation coefficients between $\overline{\dot{\omega}+\nabla \cdot(\rho D \nabla c)}$ and $\overline{\left(\rho S_{d}\right)}{ }_{s} \Sigma_{g e n}$ for both FUREBY and KEPPELER models decrease with increasing values 
of $\Delta$. The introduction of the damping factor $Q_{A}$ reduces the correlation coefficients between $\overline{\dot{\omega}+\nabla \cdot(\rho D \nabla c)}$ and $Q_{A}{\overline{\left(\rho S_{d}\right)}}_{s} \Sigma_{g e n}$ for both the FUREBY and KEPPELER models.

The KEPPELER model exhibits higher correlation coefficients between $\overline{\dot{\omega}+\nabla \cdot(\rho D \nabla c)}$ and $Q_{A} \overline{\left(\rho S_{d}\right)_{S}} \Sigma_{g e n}$ than for the FUREBY model for $\Delta>\delta_{t h}$ for case A at all times shown in Fig. 7. A qualitatively similar trend has been observed until $t=6 \delta_{Z} / S_{L}$ for all filter widths in cases C and E. By contrast, the FUREBY model shows either almost the same or slightly greater correlation coefficient between $\overline{\dot{\omega}+\nabla \cdot(\rho D \nabla c)}$ and $Q_{A} \overline{\left(\rho S_{d}\right)}{ }_{s} \Sigma_{g e n}$ than the KEPPELER model for $\Delta=2.8 \delta_{t h}$. The presence of $Q_{A}$ reduces the correlation coefficient between $\overline{\dot{\omega}+\nabla \cdot(\rho D \nabla c)}$ and $Q_{A}{\overline{\left(\rho S_{d}\right)}}_{s} \Sigma_{g e n}$ for both FUREBY and KEPPELER models.

It can further be seen from Fig. 7 that the correlation coefficients between $\overline{\dot{\omega}+\nabla \cdot(\rho D \nabla c)}$ and $\rho_{0} S_{L} \Sigma_{\text {gen }}$ for both FUREBY and KEPPELER models follow the same qualitative trends as the corresponding correlation coefficients between $\overline{\dot{\omega}+\nabla \cdot(\rho D \nabla c)}$ and $\overline{\left(\rho S_{d}\right)_{s}} \Sigma_{g e n}$. However, the magnitude of the correlation coefficients between $\overline{\dot{\omega}+\nabla \cdot(\rho D \nabla c)}$ and $\rho_{0} S_{L} \Sigma_{g e n}$ is found to be significantly smaller than the correlation coefficients between $\overline{\dot{\omega}+\nabla \cdot(\rho D \nabla c)}$ and $\overline{\left(\rho S_{d}\right)_{s}} \Sigma_{\text {gen }}$ before flame quenching. This is because of the fact that the local behaviour of ${\overline{\left(\rho S_{d}\right)}}_{s}$ such as its strain rate and curvature dependence is not sufficiently captured by $\rho_{0} S_{L}$, which leads to a smaller correlation coefficient between $\overline{\dot{\omega}+\nabla \cdot(\rho D \nabla c)}$ and $\rho_{0} S_{L} \Sigma_{g e n}$ than the corresponding value obtained for $\overline{\dot{\omega}+\nabla \cdot(\rho D \nabla c)}$ and $\overline{\left(\rho S_{d}\right)_{s}} \Sigma_{g e n}$. This behaviour is evident for both FUREBY and KEPPELER models irrespective of their evaluation techniques, which is consistent with recent findings by Klein et al. (2016). Furthermore, both $Q_{A} \rho_{0} S_{L} \Sigma_{g e n}$ and $Q_{B} \rho_{0} S_{L} \Sigma_{g e n}$ yield significantly smaller correlation coefficients with $\overline{\dot{\omega}+\nabla \cdot(\rho D \nabla c)}$ than the corresponding value for the correlation between $\overline{\dot{\omega}+\nabla \cdot(\rho D \nabla c)}$ and $\rho_{0} S_{L} \Sigma_{g e n}$. 
Furthermore, negative correlations between $\overline{\dot{\omega}+\nabla \cdot(\rho D \nabla c)}$ and $\rho_{0} S_{L} \Sigma_{\text {gen }}$ for both the FUREBY and KEPPELER models can be obtained at an advanced stage of flame quenching (e.g. at $t=10 \delta_{Z} / S_{L}$ for case $\mathrm{E}$ ) and same is true for both $Q_{A} \rho_{0} S_{L} \Sigma_{g e n}$ and $Q_{B} \rho_{0} S_{L} \Sigma_{g e n}$. It can further be seen from Fig. 7 that the correlation coefficient between $\overline{\dot{\omega}+\nabla \cdot(\rho D \nabla c)}$ and $Q_{j} \rho_{0} S_{L} \Sigma_{\text {gen }}$ (see eq. 14) remains comparable to the correlation coefficient between $\overline{\dot{\omega}+\nabla \cdot(\rho D \nabla c)}$ and $\overline{\left(\rho S_{d}\right)_{s}} \Sigma_{g e n}$ according to the FUREBY model. The modification $Q_{j} \rho_{0} S_{L} \Sigma_{\text {gen }}$ (see eq. 14) significantly improves the correlation with $\overline{\dot{\omega}+\nabla \cdot(\rho D \nabla c)}$ especially during the advanced stage of flame quenching (e.g. $t_{5}=10 \delta_{Z} / S_{L}$ in cases $\mathrm{C}$ and $\mathrm{E}$ ).

\section{CONCLUSIONS}

Based on this analysis the applicability of previously proposed algebraic LES closures of FSD has been assessed using three-dimensional DNS data for head-on quenching of premixed turbulent flames on isothermal inert walls for different values of $\mathrm{Da}$ and $\mathrm{Ka}$. Here, the algebraic FSD closure proposed by Fureby (2005) has been considered for this a-priori analysis alongside a model proposed by Keppeler et al. $(2012,2014)$ which has recently been used for LES of flame-wall interaction. The applicability of wall correction factors, which were originally proposed in the context of RANS, has also been assessed for LES based on the current a-priori DNS analysis. Both the models underpredict the generalised FSD in the nearwall region for all cases considered here, and the use of the RANS based wall correction factor worsens the (under)prediction of FSD in the near-wall region. By contrast, $\rho_{0} S_{L} \Sigma_{g e n}$ overpredicts $\overline{\dot{\omega}+\nabla \cdot(\rho D \nabla c)}$ in the near-wall region for both FSD models. Although $\rho_{0} S_{L} \Sigma_{g e n}$ extracted from DNS data accurately captures the mean behaviour of $\overline{\dot{\omega}+\nabla \cdot(\rho D \nabla c)}$ away from the wall, the correlation between these quantities remains small because $\rho_{0} S_{L}$ does not sufficiently capture the local behaviour of $\overline{\left(\rho S_{d}\right)}$ s for all cases considered here. The use of an 
existing wall damping factor, which was originally proposed for RANS, yields vanishingly small values of $\overline{\dot{\omega}+\nabla \cdot(\rho D \nabla c)}$ close to the wall even when the combined reaction-diffusion term assumes significant values in the near-wall region. Based on this analysis, near-wall modifications for FSD $\Sigma_{g e n}$ and the reaction-diffusion imbalance term $\overline{\dot{\omega}+\nabla \cdot(\rho D \nabla c)}$ have been proposed, which have been shown to accurately capture the corresponding terms extracted from DNS data. An accurate closure of reaction-diffusion imbalance term enables high-fidelity conjugate heat transfer calculations which yield not only a precise prediction of the temperature field for the combusting gases but also for the combustor walls. Thus, the closure of reactiondiffusion imbalance term can play a pivotal role in the design-cycle of combustors which are increasingly made smaller in size due to compactness and also for the sake of increasing energy density.

Although the corrections proposed in this analysis perform better than the ones suggested earlier (Alshaalan et al., 1998; Bruneaux et al., 1996), and capture the desired asymptotic behaviours by incorporating the information regarding the quenching distance (in the form of minimum Peclet number) and enthalpy loss (in terms of $(\tilde{c}-\tilde{T})$ ), their functional forms involve a degree of empiricism. Thus, further investigation will be needed for validation of the FSD based closures in the near-wall region for other flame quenching configurations in the presence of detailed chemistry and transport.

\section{ACKNOWLEDGEMENTS}

The authors are grateful to N8, ARCHER and EPSRC for computational support. 


\section{REFERENCES}

Allauddin, U., Pfitzner, M., Klein, M., Chakraborty, N. 2017.A-priori and a-posteriori analysis of algebraic flame surface density modelling in the context of large eddy simulation of turbulent premixed combustion. Numer. Heat Trans. A,

Alshaalan, T.M., Rutland, C.J. 1998. Turbulence, scalar transport, and reaction rates in flamewall interaction. Proc. Combust. Inst., 27, 793.

Alshaalan, T., Rutland, C.J. 2002. Wall heat flux in turbulent premixed reacting flow. Combust. Sci. Technol., 174, 135.

Boger, M., Veynante, D., Boughanem, H., Trouvé, A. 1998. Direct Numerical Simulation analysis of flame surface density concept for Large Eddy Simulation of turbulent premixed combustion. Proc. Combust. Inst. 27, 917.

Butz, D., Gao, Y., Kempf, A.M., Chakraborty, N. 2015. Large Eddy Simulations of a turbulent premixed swirl flame using an algebraic Scalar Dissipation Rate closure, Combust. Flame, 162, 3180.

Bruneaux, G., Akselvoll, K., Poinsot, T., Ferziger, J. 1996. Flame-wall interaction simulation in a turbulent channel flow. Combust. Flame, 107, 27.

Bruneaux, G., Poinsot, T., Ferziger, J. 1997. Premixed flame-wall interaction in a turbulent channel flow: budget for the flame surface density evolution equation and modelling. J. Fluid Mech., 349, 191.

Candel, S.M., Poinsot, T.J. 1990. Flame stretch and the balance equation for the flame area, Combust. Sci. Technol., 70,1.

Candel, S., Veynante, D., Lacas, F., Maistret, E., Darabhia, N., Poinsot, T. 1990. Coherent Flamelet Model: Applications and recent extensions, in Recent Advances in Combustion Modelling, ed. B.E. Larrouturou, World Scientific, Singapore, pp. 19-64. 
Cant, R.S., Bray, K.N.C. 1988. Strained laminar flamelet calculations of premixed turbulent combustion in a closed vessel, Proc. Combust. Inst., 22, 791.

Cant, R.S., Pope, S.B., Bray, K.N.C. 1990. Modelling of flamelet surface to volume ratio in turbulent premixed combustion, Proc. Combust. Inst., 23, 809.

Chakraborty, N., Cant, R. S. 2007. A priori analysis of the curvature and propagation terms of the flame surface density transport equation for large eddy simulation. Phys. Fluids 19, 105101. Chakraborty, N., Klein, M. 2008. A-priori direct numerical simulation assessment of algebraic flame surface density models for turbulent premixed flames in the context of large eddy simulation. Phys. Fluids 20, 085108.

Chakraborty, N., Cant, R. S. 2009. Direct Numerical Simulation analysis of the Flame Surface Density transport equation in the context of Large Eddy Simulation. Proc. Combust. Inst., 32, 1445.

Chakraborty, N., Cant, R.S. 2011. Effects of Lewis number on Flame Surface Density transport in turbulent premixed combustion, Combust. Flame 158, 1768.

Charlette, F., Meneveau, C., Veynante, D. 2002. A power law wrinkling model for LES of premixed turbulent combustion, Part I: Non dynamic formulation and initial tests, Combust. Flame, 131, 159.

Chen, J.H., Choudhary, A., De Supinski, B., DeVries, M., Hawkes, E., Klasky, S., Liao, W., Ma, K., Mellor-Crummey, J., Podhorszki, N., Sankaran, R., Shende, S., Yoo, C. 2009. Terascale direct numerical simulations of turbulent combustion using s3d. Comput. Sci. \& Discov., 2, 015001.

Dabireau, F., Cuenot, B., Vermorel, O., Poinsot, T. 2013. Interaction of flames of $\mathrm{H} 2+\mathrm{O} 2$ with inert walls. Combust. Flame, 135, 123.

Fureby, C. 2005. A fractal flame-wrinkling large eddy simulation model for premixed turbulent combustion, Proc. Combust. Inst. 30, 593. 
Gruber, A., Sankaran, R., Hawkes, E., Chen, J. 2010. Turbulent flame-wall interaction: a direct numerical simulation study. J Fluid Mech., 658, 5.

Gruber, A., Chen, J. H., Valiev, D., Law, C.K. 2012. Direct numerical simulation of premixed flame boundary layer flashback in turbulent channel flow. J. Fluid Mech., 709, 516.

Hernandez-Perez, F.E., Yuen, F. T. C., Groth, C.P.T., Gülder, Ö. L. 2011. LES of a laboratoryscale turbulent premixed Bunsen flame using FSD, PCM-FPI and thickened flame models, Proc. Combust. Inst., 33, 1365.

Hawkes, E.R., and Cant, R.S. 2000. A flame surface density approach to large eddy simulation of premixed turbulent combustion, Proc. Combust. Inst., 28, 51.

Hawkes, E.R., Cant, R.S. 2001. Implications of a flame surface density approach to large eddy simulation of premixed turbulent combustion. Combust. Flame 126, 1617.

Heywood, J.B. 1988. Internal combustion engine fundamentals, McGraw-Hill, New York. Huang, W.M., Vosen, S.R., Greif, R. 1986. Heat transfer during laminar flame quenching, Proc. Combust. Inst. 21, 1853.

Jarosinsky, J. 1986. A survey of recent studies on flame extinction, Combust. Sci. Technol. 12, 81.

Jenkins, K.W., Cant, R.S. 1999. Direct numerical simulation of turbulent flame kernels. In: Recent Advances in DNS and LES, 191-202. Springer.

Katragadda, M., Chakraborty, N., Cant, R.S. 2012a. A-priori DNS assessment of wrinkling factor based algebraic Flame Surface Density models in the context of Large Eddy Simulations for non-unity Lewis number flames in the thin reaction zones regime, J. Combust., 794671. Katragadda, M., Chakraborty, N., Cant, R.S. 2012b Effects of turbulent Reynolds number on the performance of algebraic Flame Surface Density models for Large Eddy Simulation in the thin reaction zones regime: A Direct Numerical Simulation analysis, J. Combust., 353257. 
Katragadda, M., Gao, Y., Chakraborty, N. 2014. Modelling of the strain rate contribution to the FSD transport for non-unity Lewis number flames in LES, Combust. Sci. Technol., 186, 1338.

Kempf, A.M., Wysocki, S., Pettit, M. 2012. An efficient, parallel low-storage implementation of Klein's turbulence generator for LES and DNS, Comput. Fluids 60, 58.

Keppeler, R., Tangermann, E., Allaudin, U., Pfitzner, M. (2014) LES of Low to High Turbulent Combustion in an Elevated Pressure Environment. Flow Turb. Combust. 92, 767.

Keppeler, R., Pfitzner, M., Tay-Wo-Chong, L., Komarek, T., Polifke, W. 2012. Including heat loss and quench effects in algebraic models for large eddy simulation of premixed combustion. Proceedings of ASME Turbo Expo 2012, GT2012-68689, Copenhagen, Denmark.

Klein, M., Chakraborty, N., Pfitzner, M. 2016. Analysis of the combined modelling of subgrid transport and filtered flame propagation for premixed turbulent combustion, Flow Turb. Combust., 96, 921.

Lai, J., Chakraborty, N. 2016a. Effects of Lewis Number on Head on Quenching of Turbulent Premixed Flame: A Direct Numerical Simulation analysis, Flow Turb. Combust., 96, 279.

Lai, J., Chakraborty, N. 2016b. Statistical behaviour of scalar dissipation rate for head on quenching of turbulent premixed flames: A Direct Numerical Simulation analysis, Combust. Sci. Technol., 188,250.

Lai, J., Chakraborty, N. 2016c. A-priori Direct Numerical Simulation modelling of Scalar Dissipation Rate transport in head-on quenching of turbulent premixed flames, Combust. Sci. Technol., 188, 1440.

Lai, J., Moody, A., Chakraborty, N. 2017. Turbulent kinetic energy transport in head-on quenching of turbulent premixed flames in the context of Reynolds Averaged Navier Stokes simulations, Fuel, doi.org/10.1016/j.fuel.2017.02.091. 
Liu, S., Meneveau, C., Katz, J. 1994. On the properties of similarity subgrid scale models as deduced from measurements in a turbulent jet. J. Fluid. Mech. 275, 83.

Ma, T., Stein, T.O., Chakraborty, N., Kempf, A.M. 2013. A posteriori testing of algebraic flame surface density models for LES. Combust. Theor. Modell. 17, 431.

Ma, T., Stein, T.O., Chakraborty, N., Kempf, A.M. 2014a. A-posteriori testing of the Flame Surface Density transport equation for LES, Combust. Theor. Modell., 18, 32.

Ma, T., Gao, Y., Kempf, A,M., Chakraborty, N. 2014b. Validation and Implementation of algebraic LES modelling of Scalar Dissipation Rate for reaction rate closure in turbulent premixed combustion, Combust. Flame, 161, 3134.

Poinsot, T., Lele, S.K. 1992. Boundary conditions for direct simulation of compressible viscous flows, J. Comp. Phys., 101, 104.

Poinsot, T., Veynante, D. 2001. Theoretical and numerical combustion, R.T. Edwards, New York.

Poinsot, T., Haworth, D., Bruneaux, G. 1993. Direct simulation and modeling of flame-wall interaction for premixed turbulent combustion. Combust. Flame. 95, 118.

Reddy, H., Abraham, J. 2012. Two-Dimensional Direct Numerical Simulation Evaluation of the Flame Surface Density Model for Flames Developing from an Ignition Kernel in Lean Methane/Air Mixtures Under Engine Conditions, Phys. Fluids, 24,105108.

Rogallo, R.S. 1981. Numerical experiments in homogeneous turbulence, NASA Technical Memorandum 81315, NASA Ames Research Center, California.

Vosen, S.R., Greif, R., Westbrook, C. 1984. Unsteady heat transfer in laminar quenching, Proc. Combust. Inst. 20, 76.

Wray, A.A. 1990. Minimal storage time advancement schemes for spectral methods, unpublished report, NASA Ames Research Center, California. 


\section{TABLES}

\begin{tabular}{cccccc}
\hline Case & $\mathrm{A}$ & $\mathrm{B}$ & $\mathrm{C}$ & $\mathrm{D}$ & $\mathrm{E}$ \\
\hline$u^{\prime} / S_{L}$ & 5.0 & 6.25 & 7.5 & 9.0 & 11.25 \\
$L_{11} / \delta_{t h}$ & 1.67 & 1.44 & 2.5 & 4.31 & 3.75 \\
$D a$ & 0.33 & 0.23 & 0.33 & 0.48 & 0.33 \\
$K a$ & 8.65 & 13.0 & 13.0 & 13.0 & 19.5 \\
$R e_{t}$ & 22.0 & 23.5 & 49.0 & 100 & 110 \\
\hline
\end{tabular}

Table 1: List of initial turbulence parameters away from the wall.

\begin{tabular}{ccccccccccc}
\hline & \multicolumn{2}{c}{ Case A } & \multicolumn{2}{c}{ Case B } & \multicolumn{2}{c}{ Case C } & \multicolumn{2}{c}{ Case D } & \multicolumn{2}{c}{ Case E } \\
\hline$t S_{L}$ & $A_{T} / A_{L}$ & $S_{T} / S_{L}$ & $A_{T} / A_{L}$ & $S_{T} / S_{L}$ & $A_{T} / A_{L}$ & $S_{T} / S_{L}$ & $A_{T} / A_{L}$ & $S_{T} / S_{L}$ & $A_{T} / A_{L}$ & $S_{T} / S_{L}$ \\
\hline$/ \delta_{Z}$ & & & & & & & & & & \\
\hline 1 & 1.57 & 1.55 & 1.59 & 1.57 & 2.79 & 2.78 & 2.91 & 2.90 & 4.18 & 4.17 \\
2 & 1.67 & 1.66 & 1.60 & 1.59 & 2.99 & 2.99 & 3.95 & 3.93 & 4.77 & 4.76 \\
4 & 1.77 & 1.76 & 1.73 & 1.71 & 2.34 & 2.31 & 2.39 & 2.37 & 2.03 & 2.01 \\
6 & 1.64 & 1.62 & 1.68 & 1.66 & 1.43 & 1.41 & 0.74 & 0.74 & 0.68 & 0.67 \\
8 & 1.51 & 1.49 & 1.53 & 1.51 & 0.50 & 0.49 & 0.20 & 0.19 & 0.12 & 0.12 \\
10 & 0.86 & 0.85 & 0.85 & 0.84 & 0.09 & 0.09 & 0.03 & 0.03 & 0.03 & 0.03 \\
\hline
\end{tabular}

Table 2: List of normalised flame surface area $A_{T} / A_{L}$ and turbulent flame speed $S_{T} / S_{L}$ at different stages of flame quenching for all cases considered here. 


\section{FIGURE CAPTIONS}

Figure 1: Snapshots of reaction progress variable $c$ and non-dimensional temperature $T$ at nondimensional time $t_{1}=2 \delta_{Z} / S_{L}, t_{2}=4 \delta_{Z} / S_{L}, t_{3}=6 \delta_{Z} / S_{L}, t_{4}=8 \delta_{Z} / S_{L}, t_{5}=10 \delta_{Z} / S_{L}$ for turbulent case $\mathrm{E}$ and its temporal evolutions of the maximum, mean and minimum values of Peclet number $P e$ (based on $T=0.9$ isosurface) and non-dimensional wall heat flux $\Phi$ together with the corresponding laminar values.

Figure 2: Variations of $\left\langle\Sigma_{g e n}\right\rangle \times \delta_{Z}(-)$ with $x_{1} / \delta_{Z}$ at three different time instants $t_{1}=$ $2 \delta_{Z} / S_{L}, t_{3}=6 \delta_{Z} / S_{L}, t_{5}=10 \delta_{Z} / S_{L}$ (1st row to 3rd row for each turbulent case) obtained from DNS data along with the predictions of the FUREBY (- - KEPPELER ( $\longrightarrow$ ) models and Eq. $12(" * ")$ evaluated based on the DNS grid spacing. The predictions of $\left\langle Q_{A} \Sigma_{g e n}\right\rangle \times \delta_{Z}$ according to Alshaalan and Rutland (1998) damping factor are also shown for the FUREBY (" + ") and KEPPELER (" + ") model evaluations using the DNS grid spacing.

Figure 3: Variations of $\left\langle\Sigma_{g e n}\right\rangle \times \delta_{Z}(-)$ with $x_{1} / \delta_{Z}$ at three different time instants $t_{1}=$ $2 \delta_{Z} / S_{L}, t_{3}=6 \delta_{Z} / S_{L}, t_{5}=10 \delta_{Z} / S_{L}$ (1st row to 3 rd row for each turbulent case) obtained from DNS data along with the predictions of the FUREBY (- - KEPPELER ( - ) models and Eq. $13(-\cdots)$ evaluated based on the LES grid spacing. The predictions of $\left\langle Q_{A} \Sigma_{g e n}\right\rangle \times \delta_{Z}$ according to Alshaalan and Rutland (1998) damping factor are also shown for the FUREBY ($\left.+{ }^{+}\right)$and KEPPELER $\left({ }^{+}+{ }^{-}\right)$model evaluations using the LES grid spacing.

Figure 4: Variations of the correlation coefficient between $\Sigma_{g e n}$ from DNS data and the predictions of the models: FUREBY —, KEPPELER —, FUREBY with Alshaalan and Rutland (1998) damping factor — KEPPELER with Alshaalan and Rutland (1998) damping factor $\longrightarrow$ and New model (Eq. 12 for (a) and Eq. 13 for (b)) - at $t_{1}=2 \delta_{Z} / S_{L}$, $t_{3}=6 \delta_{Z} / S_{L}, t_{5}=10 \delta_{Z} / S_{L}$ for (a) DNS grid spacing and (b) LES grid spacing based evaluations. 
Figure 5: Variations of $\langle\overline{\dot{\omega}+\nabla \cdot(\rho D \nabla c)}\rangle \times \delta_{Z} / \rho_{0} S_{L}(\longrightarrow)$ and $\left\langle\rho_{0} S_{L} \Sigma_{g e n}\right\rangle \times \delta_{Z} / \rho_{0} S_{L}(\cdots \cdot \cdot)$ with $x_{1} / \delta_{Z}$ at three different time instants $t_{1}=2 \delta_{Z} / S_{L}, t_{3}=6 \delta_{Z} / S_{L}, t_{5}=10 \delta_{Z} / S_{L} \quad(1 \mathrm{st}$ row to 3rd row for each turbulent case) obtained from DNS data along with the FUREBY (—), KEPPELER (—) model predictions evaluated based on the DNS grid spacing. The predictions of $\left\langle Q_{A} \rho_{0} S_{L} \Sigma_{g e n}\right\rangle \times \delta_{Z} / \rho_{0} S_{L}$ (shown with + ) and $\left\langle Q_{B} \rho_{0} S_{L} \Sigma_{g e n}\right\rangle \times \delta_{Z} / \rho_{0} S_{L}$ (shown with o) according to Alshaalan and Rutland (1998) and Bruneaux et al. (1997) damping factor are shown according to the FUREBY(shown in red) and KEPPELER (shown in blue) model evaluations using the DNS grid spacing. The predictions of $\left\langle Q_{J} \rho_{0} S_{L} \Sigma_{g e n}\right\rangle \times \delta_{Z} / \rho_{0} S_{L}$ (see Eq. 14) for the DNS grid spacing are shown by ("n"*).

Figure 6: Variations of $\langle\overline{\dot{\omega}+\nabla \cdot(\rho D \nabla c)}\rangle \times \delta_{Z} / \rho_{0} S_{L}(\longrightarrow)$ and $\left\langle\rho_{0} S_{L} \Sigma_{g e n}\right\rangle \times \delta_{Z} / \rho_{0} S_{L}(\cdots \cdot \cdot)$ with $x_{1} / \delta_{Z}$ at three different time instants $t_{1}=2 \delta_{Z} / S_{L}, t_{3}=6 \delta_{Z} / S_{L}, t_{5}=10 \delta_{Z} / S_{L} \quad(1 \mathrm{st}$ row to 3rd row for each turbulent case) obtained from DNS data along with the FUREBY (—), KEPPELER (—) model predictions evaluated based on the LES grid spacing. The predictions of $\left\langle Q_{A} \rho_{0} S_{L} \Sigma_{g e n}\right\rangle \times \delta_{Z} / \rho_{0} S_{L}$ (shown with + ) and $\left\langle Q_{B} \rho_{0} S_{L} \Sigma_{g e n}\right\rangle \times \delta_{Z} / \rho_{0} S_{L}$ (shown with o) according to Alshaalan and Rutland (1998) and Bruneaux et al. (1997) damping factor are shown according to the FUREBY(shown in red) and KEPPELER (shown in blue) model evaluations using the LES grid spacing. The predictions of $\left\langle Q_{J} \rho_{0} S_{L} \Sigma_{g e n}\right\rangle \times \delta_{Z} / \rho_{0} S_{L}$ (see Eq. 14) for the LES grid spacing are shown by ("*"*).

Figure 7: Variations of the correlation coefficient between $\overline{\dot{\omega}+\nabla \cdot(\rho D \nabla c)}$ from DNS data and (i) $\overline{\left(\rho S_{d}\right)} \Sigma_{s} \Sigma_{g e n}$ according to the FUREBY - KEPPELER - models, (ii) $Q_{A} \overline{\left(\rho S_{d}\right)}{ }_{s} \Sigma_{g e n}$ according to the FUREBY — KEPPELER — models, (iii) $\rho_{0} S_{\mathrm{L}} \Sigma_{\text {gen }}$ according to DNS data $\longrightarrow$, FUREBY $\longrightarrow$ KEPPELER - models, (iv) $Q_{A} \rho_{0} S_{\mathrm{L}} \Sigma_{\text {gen }}$ according to FUREBY $\longrightarrow$, KEPPELER — models, (v) $Q_{B} \rho_{0} S_{\mathrm{L}} \Sigma_{\text {gen }}$ according to FUREBY —, KEPPELER 
models and $Q_{J} \rho_{0} S_{\mathrm{L}} \Sigma_{g e n}$ (see Eq. 14) - at $t_{1}=2 \delta_{Z} / S_{L}, t_{3}=6 \delta_{Z} / S_{L}, t_{5}=10 \delta_{Z} / S_{L}$ for the LES grid spacing based evaluations. 


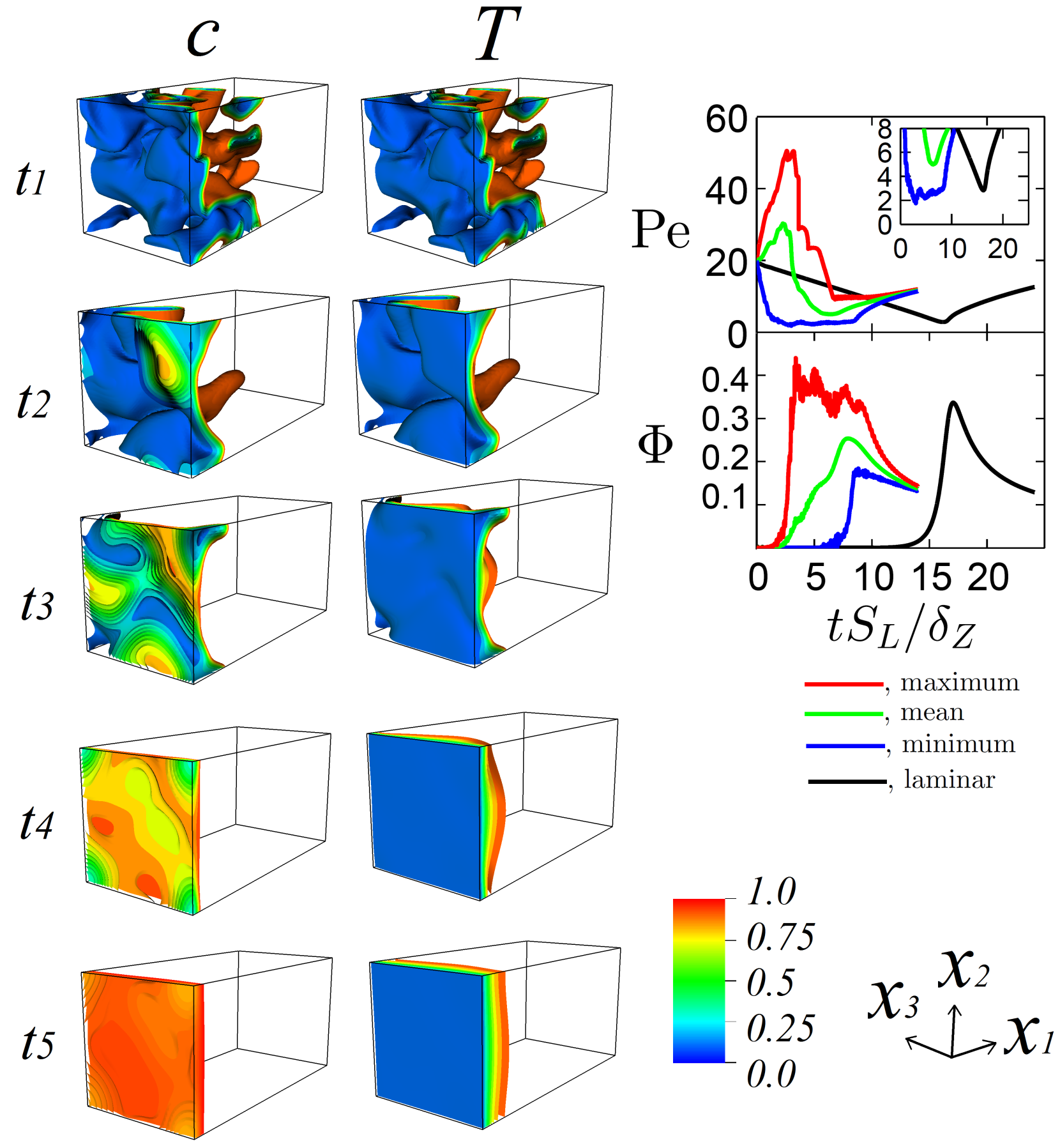

Figure 1: Snapshots of reaction progress variable $c$ and non-dimensional temperature $T$ at non-dimensional time $t_{1}=2 \delta_{Z} / S_{L}, t_{2}=4 \delta_{Z} / S_{L}, t_{3}=6 \delta_{Z} / S_{L}, t_{4}=8 \delta_{Z} / S_{L}, t_{5}=10 \delta_{Z} / S_{L}$ for turbulent case E and its temporal evolution of maximum, mean and minimum values of Peclet number $P e$ (based on $T=0.9$ isosurface) and non-dimensional wall heat flux $\Phi$ together with the corresponding laminar values. 


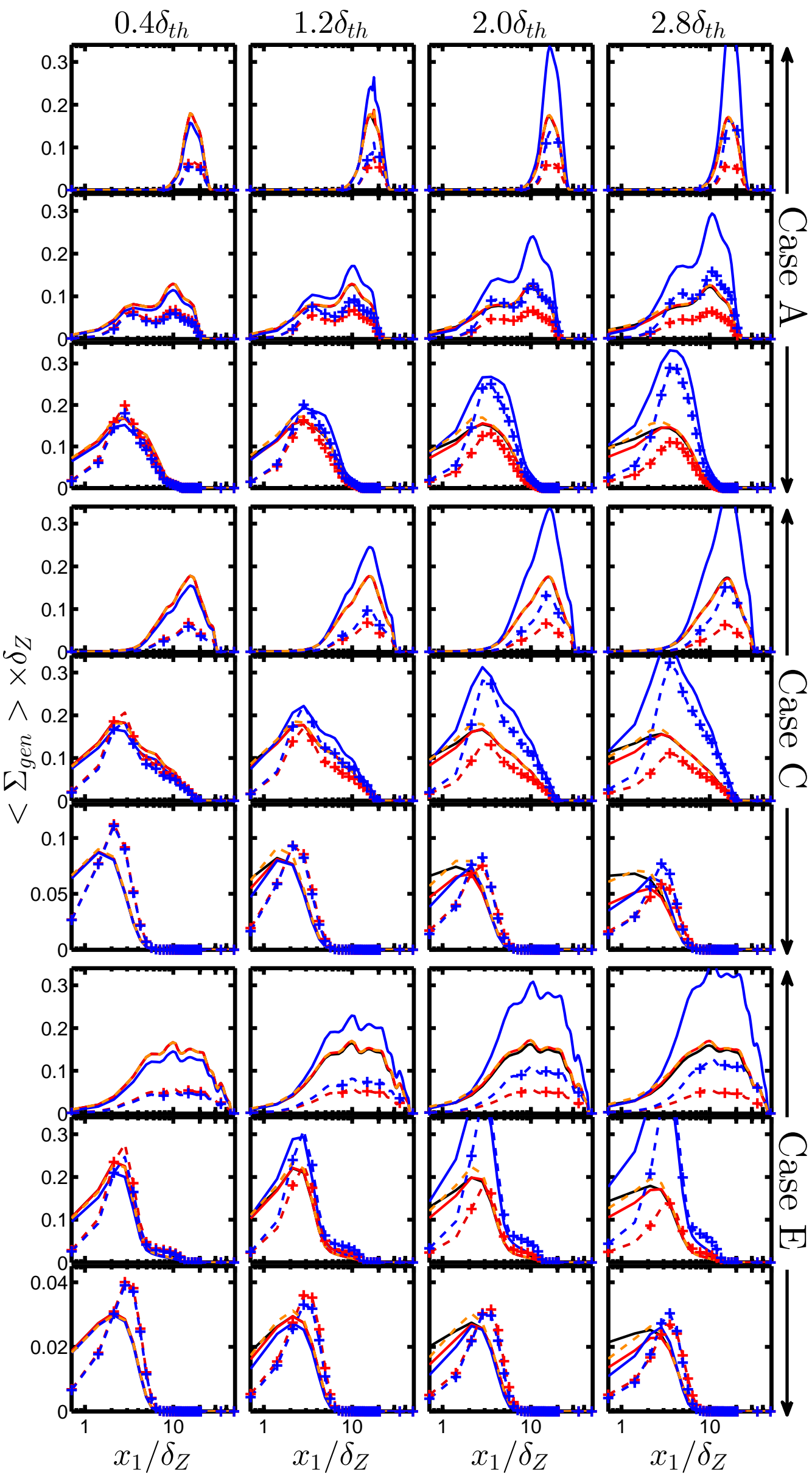

Figure 2: Variation of $\left\langle\Sigma_{g e n}\right\rangle \times \delta_{Z}(\longrightarrow)$ with $x_{1} / \delta_{Z}$ at three time instants $t_{1}=2 \delta_{Z} / S_{L}, t_{3}=6 \delta_{Z} / S_{L}$, $t_{5}=10 \delta_{Z} / S_{L}$ (1st row to 3rd row for each turbulent case) obtained from DNS data along with the FUREBY (-), KEPPELER, (- $(-)$ and Eq. $12(---)$ evaluated based on the DNS grid spacing. The predictions of $\left\langle Q_{A} \Sigma_{\text {gen }}\right\rangle \times \delta_{Z}$ according to Alshaalan and Rutland [28] damping factor are also shown for the FUREBY $(-+-)$ and KEPPELER $(-+-)$ models evaluated using the DNS grid spacing. 


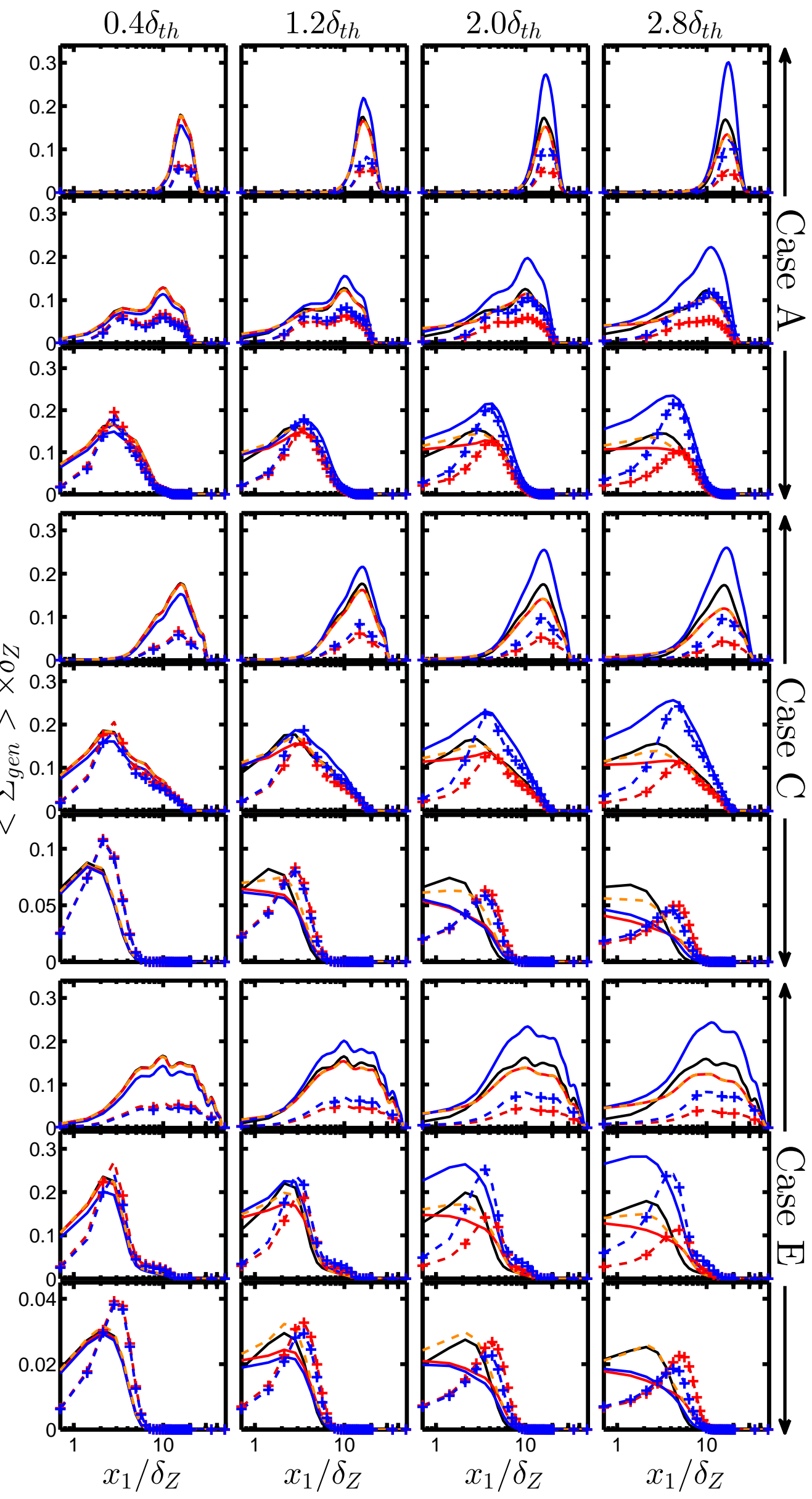

Figure 3: Variation of $\left\langle\Sigma_{g e n}>\times \delta_{Z}(\longrightarrow)\right.$ with $x_{1} / \delta_{Z}$ at three time instants $t_{1}=2 \delta_{Z} / S_{L}, t_{3}=6 \delta_{Z} / S_{L}$, $t_{5}=10 \delta_{Z} / S_{L}$ (1st row to 3rd row for each turbulent case) obtained from DNS data along with the FUREBY (-), KEPPELER, (- $(-)$ and Eq. 13 (- - ) evaluated based on the LES grid spacing. The predictions of $\left\langle Q_{A} \Sigma_{\text {gen }}\right\rangle \times \delta_{Z}$ according to Alshaalan and Rutland [28] damping factor are also shown for the FUREBY $(-+-)$ and KEPPELER $(-+-)$ models evaluated using the LES grid spacing. 


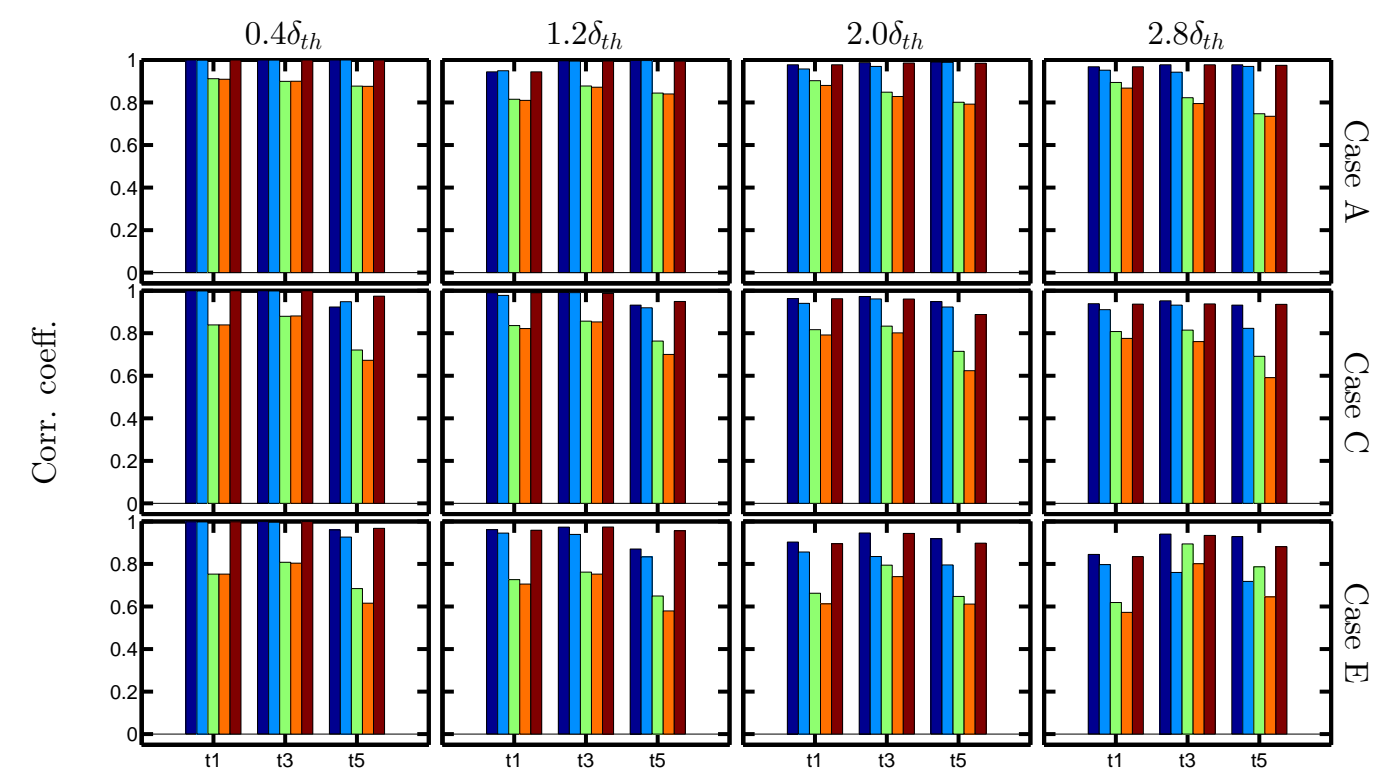

(a)

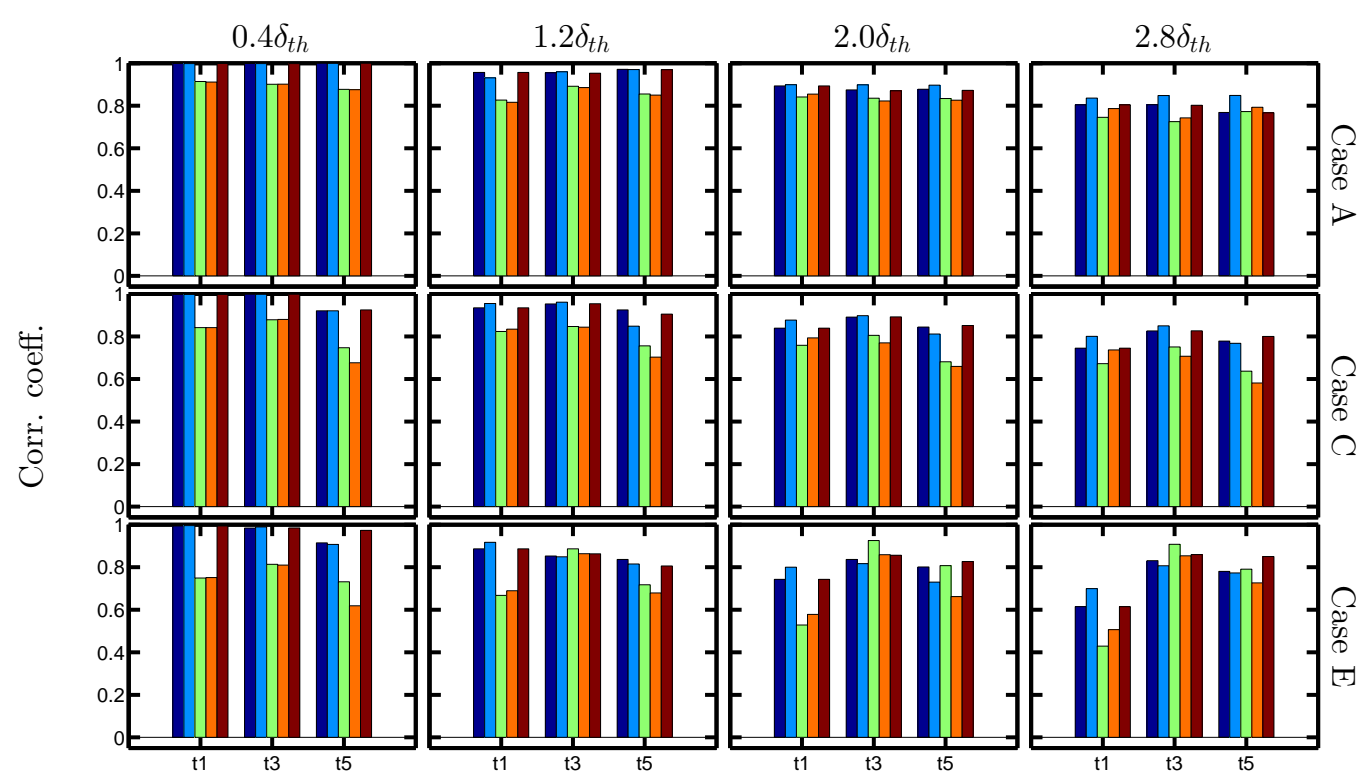

(b)

Figure 4: Variations of the correlation coefficient between $\Sigma_{g e n}$ from DNS data and the predictions of the models: FUREBY , KEPPELER , FUREBY with Alshaalan and Rutland [28] damping factor , KEPPELER with Alshaalan and Rutland [28] damping factor ${ }^{-}$and New model (Eq. 12 for (a) and Eq. 13 for (b)) at $t_{1}=2 \delta_{Z} / S_{L}, t_{3}=6 \delta_{Z} / S_{L}, t_{5}=10 \delta_{Z} / S_{L}$ for (a) DNS grid spacing and (b) LES grid spacing based evaluations. 

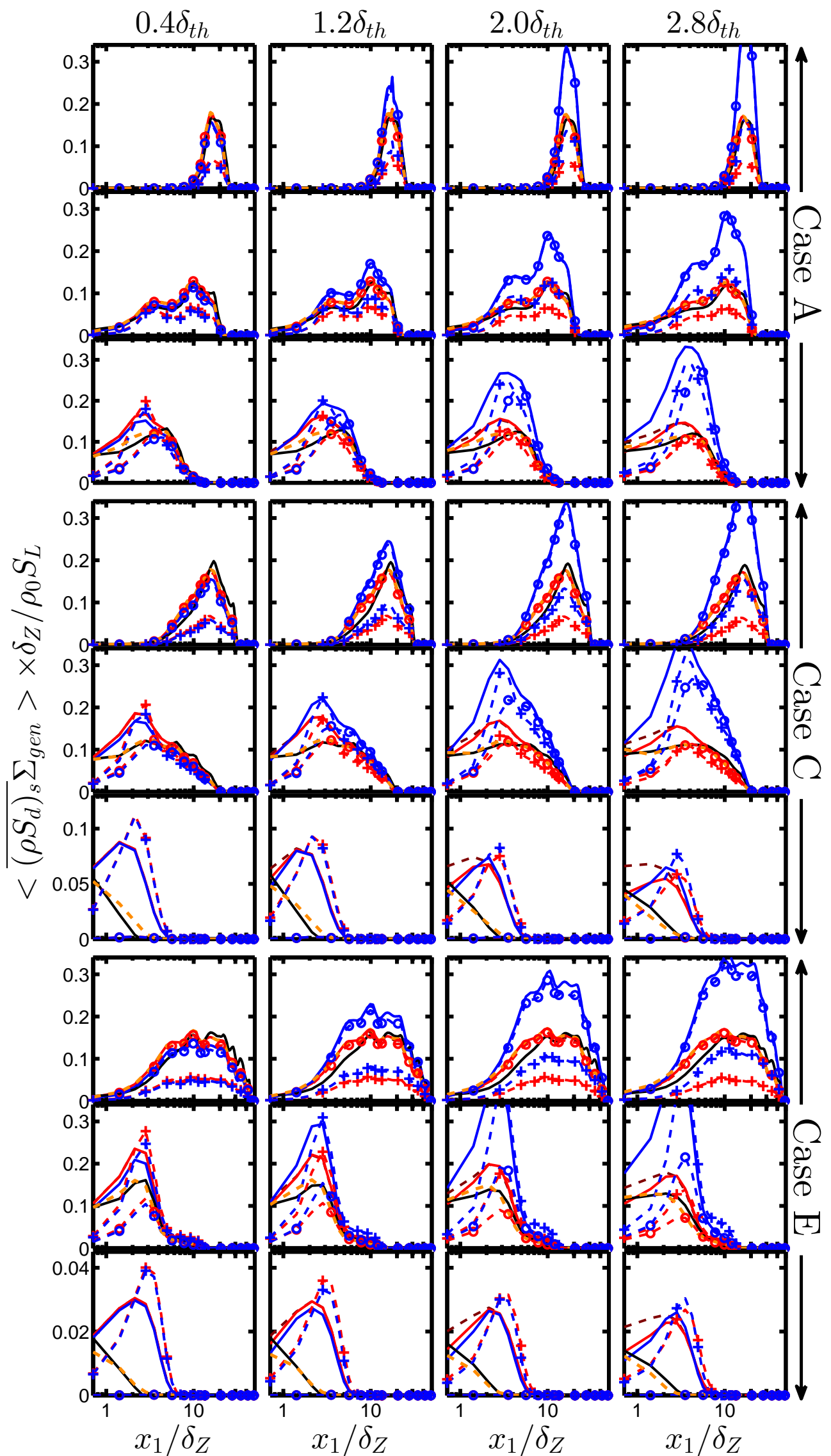

Figure 5: Variation of $\left\langle\overline{\dot{\omega}+\nabla \cdot(\rho D \nabla c)}>\times \delta_{z} / \rho_{0} S_{L}(\longrightarrow)\right.$ and $<\rho_{0} S_{L} \Sigma_{g e n}>\times \delta_{Z} / \rho_{0} S_{L}$ (- - -) with $x_{1} / \delta_{Z}$ at three different time instants $t_{1}=2 \delta_{Z} / S_{L}, t_{3}=6 \delta_{Z} / S_{L}, t_{5}=10 \delta_{Z} / S_{L}$ (1st row to 3rd row for each turbulent case) obtained from DNS data along with $<\rho_{0} S_{L} \Sigma_{\text {gen }}>\times \delta_{Z} / \rho_{0} S_{L}$ according to the FUREBY (—), KEPPELER (—) model predictions evaluated based on the DNS grid spacing. The predictions of $\left\langle Q_{A} \rho_{0} S_{L} \Sigma_{g e n}>\times \delta_{Z} / \rho_{0} S_{L}\right.$ (shown with + ) and $<Q_{B} \rho_{0} S_{L} \Sigma_{g e n}>\times \delta_{Z} / \rho_{0} S_{L}$ (shown with o) according to Alshaalan [28] and Bruneaux et al. [27] damping factor are shown according to the FUREBY (shown in red) and KEPPELER (shown in blue) model evaluations using the DNS grid spacing. The predictions of $<Q_{J} \rho_{0} S_{L} \Sigma_{g e n}>\times \delta_{Z} / \rho_{0} S_{L}$ (see Eq. 14) for the DNS grid spacing are shown by (- - -). 

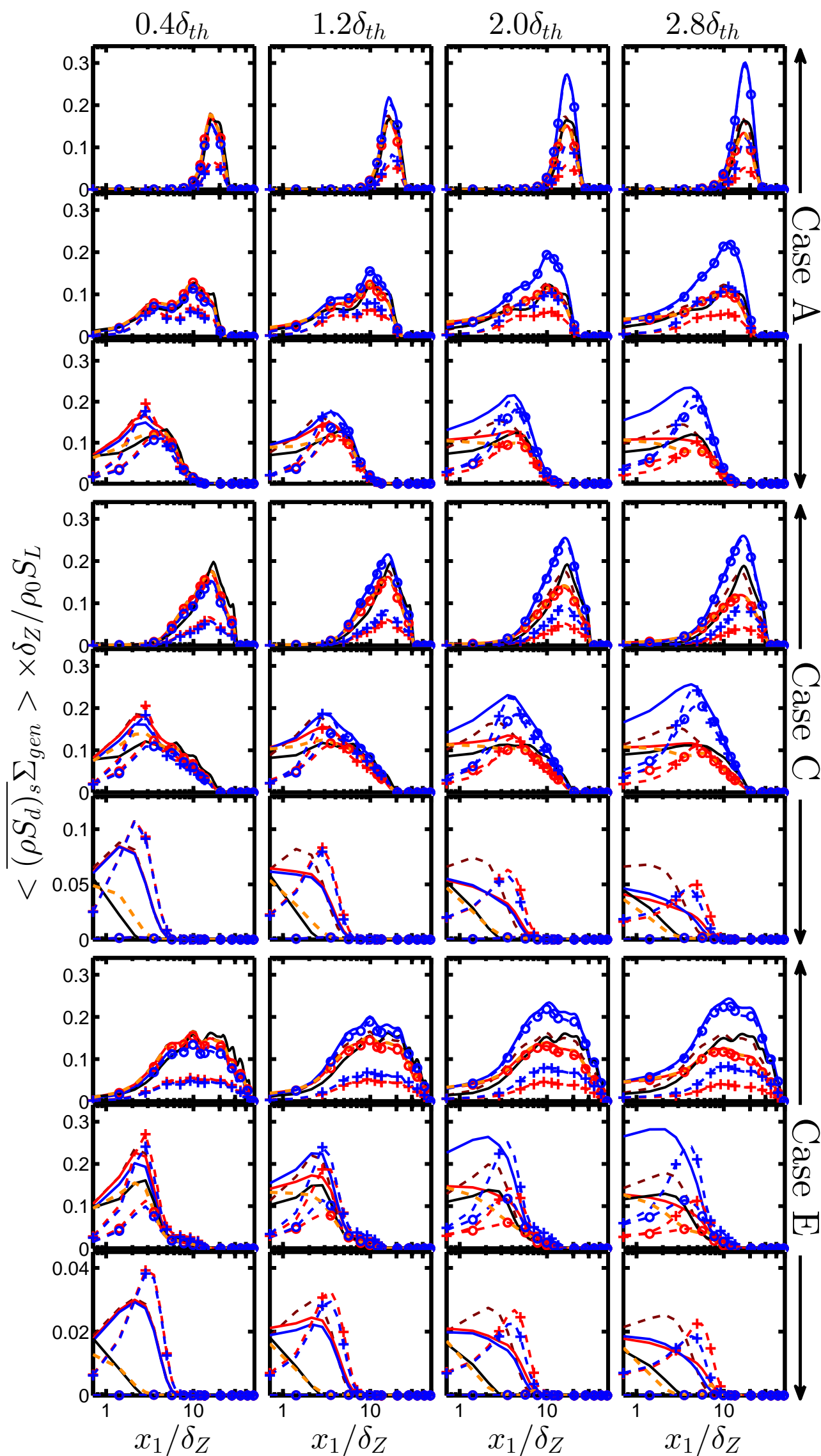

Figure 6: Variation of $\left\langle\overline{\dot{\omega}+\nabla \cdot(\rho D \nabla c)}>\times \delta_{z} / \rho_{0} S_{L}(\longrightarrow)\right.$ and $<\rho_{0} S_{L} \Sigma_{\text {gen }}>\times \delta_{Z} / \rho_{0} S_{L}(---)$ with $x_{1} / \delta_{Z}$ at three different time instants $t_{1}=2 \delta_{Z} / S_{L}, t_{3}=6 \delta_{Z} / S_{L}, t_{5}=10 \delta_{Z} / S_{L}$ (1st row to 3rd row for each turbulent case) obtained from DNS data along with $<\rho_{0} S_{L} \Sigma_{g e n}>\times \delta_{Z} / \rho_{0} S_{L}$ according to FUREBY (—), KEPPELER (-) model predictions evaluated based on the LES grid spacing. The predictions of $\left\langle Q_{A} \rho_{0} S_{L} \Sigma_{g e n}>\times \delta_{Z} / \rho_{0} S_{L}\right.$ (shown with + ) and $\left\langle Q_{B} \rho_{0} S_{L} \Sigma_{g e n}>\times \delta_{Z} / \rho_{0} S_{L}\right.$ (shown with o) according to Alshaalan [28] and Bruneaux et al. [27] damping factor are shown according to the FUREBY (shown in red) and KEPPELER (shown in blue) model evaluations using the LES grid spacing. The predictions of $<Q_{J} \rho_{0} S_{L} \Sigma_{g e n}>\times \delta_{Z} / \rho_{0} S_{L}$ (see Eq. 14) for the LES grid spacing are shown by (- - ). 


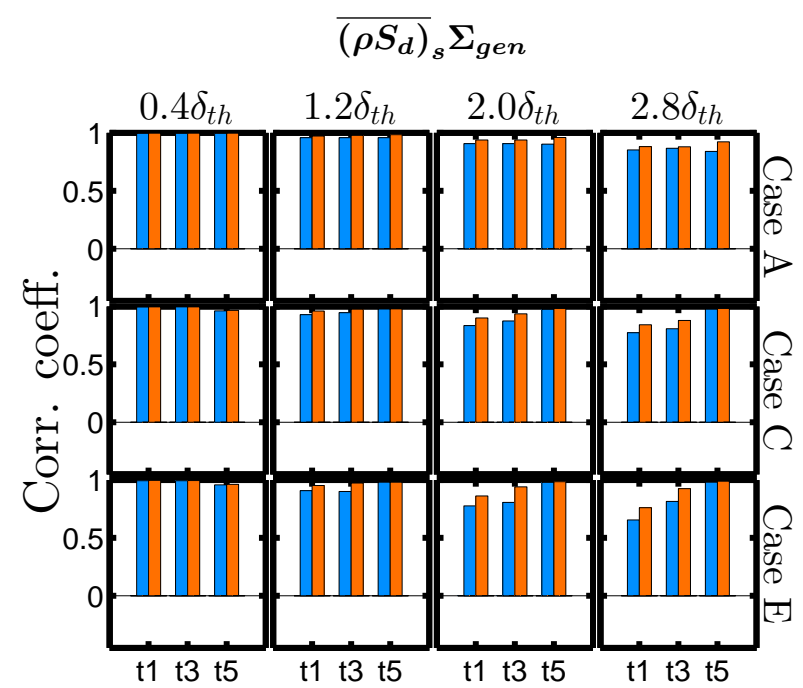

(i)

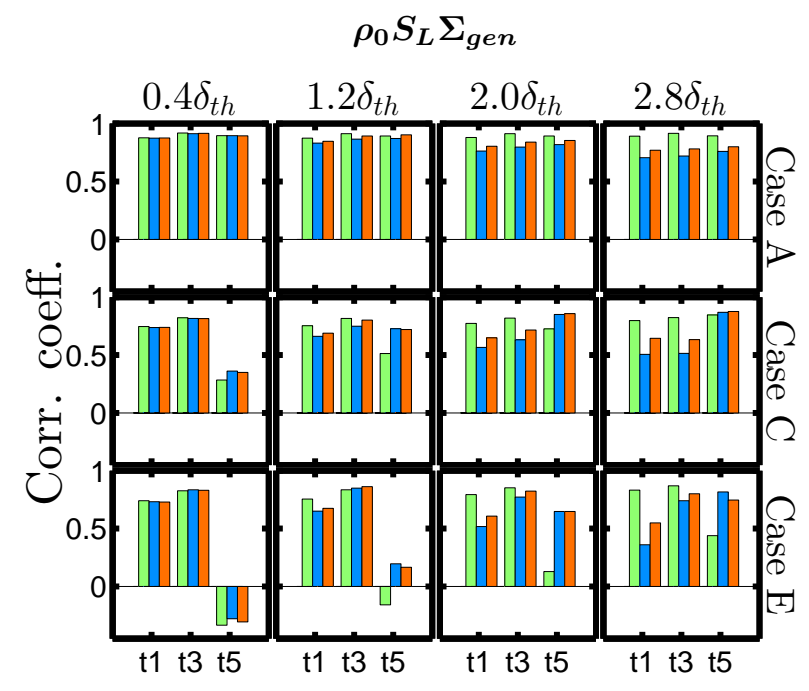

(iii)

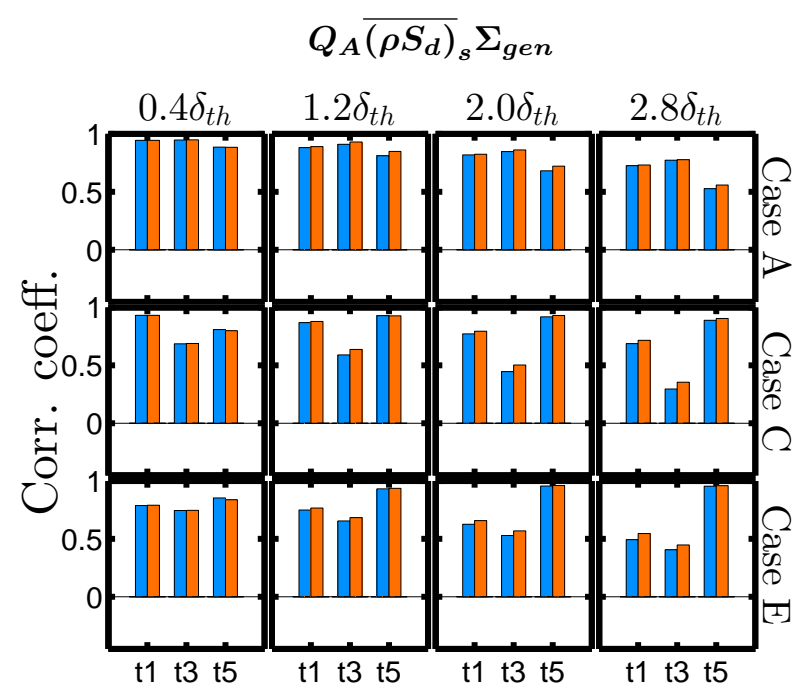

(ii)

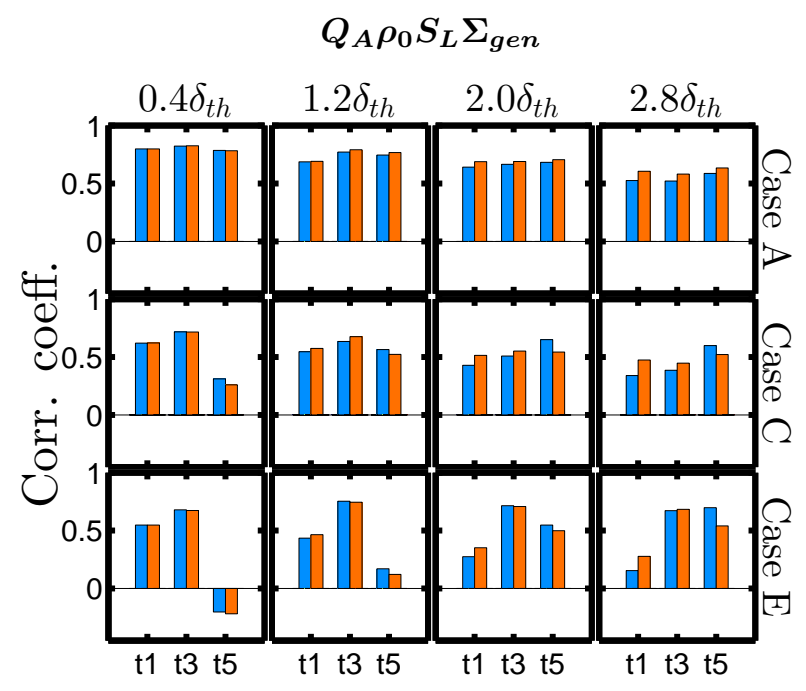

(iv)

$Q_{B} \rho_{0} S_{L} \Sigma_{g e n}$ and $Q_{j} \rho_{0} S_{L} \Sigma_{g e n}$

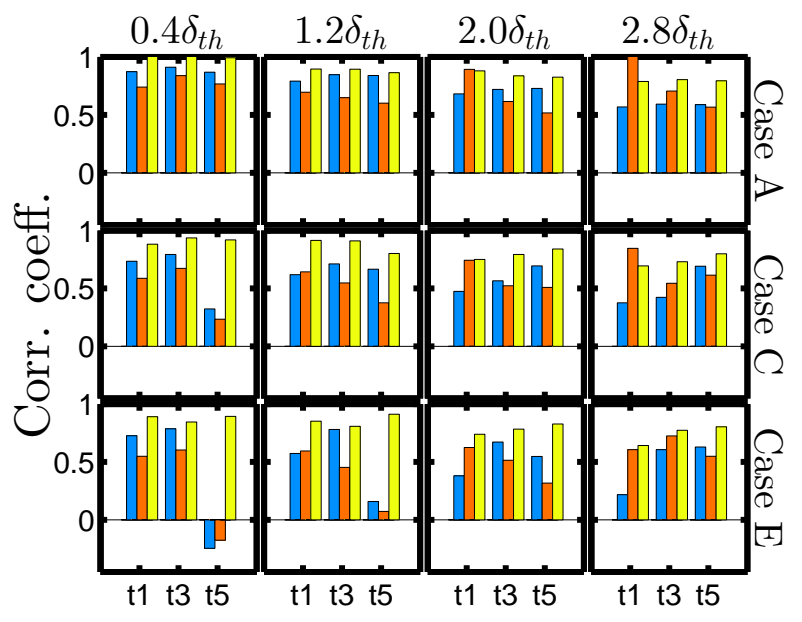

(v)

Figure 7: Variations of the correlation coefficient between $\overline{\dot{\omega}+\nabla \cdot(\rho D \nabla c)}$ from DNS data and (i) $\overline{\left(\rho S_{d}\right)_{s}} \Sigma_{g e n}$ according to the FUREBY $=$ KEPPLER models, (ii) $Q_{A} \overline{\left(\rho S_{d}\right)} \Sigma_{\text {gen }}$ according to the FUREBY , KEPPLER models, (iii) $\rho_{0} S_{L} \Sigma_{\text {gen }}$ according to the DNS data PLER models, (iv) $Q_{A} \rho_{0} S_{L} \Sigma_{\text {gen }}$ according to the FUREBY $=$, KEPPLER (v) $Q_{B} \rho_{0} S_{L} \Sigma_{\text {gen }}$ according to the FUREBY $\longrightarrow$, KEPPLER and $Q_{j} \rho_{0} S_{L} \Sigma_{\text {gen }}$ (see Eq. 14) at $t_{1}=2 \delta_{Z} / S_{L}$, $t_{3}=6 \delta_{Z} / S_{L}, t_{5}=10 \delta_{Z} / S_{L}$ for LES grid spacing based evaluations. 